$12,13,19$

\title{
Зависимость поверхностной энергии от температуры и давления для макро- и нанокристалла
}

\author{
(C) М.Н. Магомедов
}

Институт проблем геотермии и возобновляемой энергетики фрилиал объединенного института высоких температур РАН, Махачкала, Россия

E-mail: mahmag4@mail.ru

Поступила в Редакцию 7 апреля 2021 г.

В окончательной редакции 7 апреля 2021 г.

Принята к публикации 4 мая 2021 г.

На основе RP-модели нанокристалла, разработан аналитический метод расчета удельной поверхностной энергии $(\sigma)$, изохорной и изобарной производных функции $\sigma$ по температуре, и изотермических производных функции $\sigma$ по давлению и по плотности. Показано, что метод применим как для макро-, так и для нанокристалла с заданным числом атомов и с определенной формой поверхности. Для реализации этого метода параметры парного межатомного потенциала Ми-Леннард-Джонса были определены самосогласованным способом на основе термоупругих свойств кристалла. Метод был апробирован на макрокристаллах 15 однокомпонентных веществ: для 8 ГЦК-кристаллов $(\mathrm{Cu}, \mathrm{Ag}, \mathrm{Au}, \mathrm{Al}, \mathrm{Ni}, \mathrm{Rh}, \mathrm{Pd}, \mathrm{Pt})$ и для 7 ОЦК-кристаллов (Fe, V, Nb, Ta, Cr, Mo, W). Расчеты были сделаны при различных температурах и показали хорошее согласие с экспериментальными данными. На примере ГЦК-Rh изучено изменение поверхностных свойств при уменьшении размера нанокристалла вдоль изотерм 10, 300, 2000 К. Показано, что при высоких давлениях и низких температурах имеется область, где функция $\sigma$ возрастает при изоморфоизотермо-изобарическом уменьшении размера нанокристалла. С ростом температуры эта область исчезает.

Ключевые слова: поверхностная энергия, межатомный потенциал, нанокристалл, уравнение состояния, тепловое расширение.

DOI: 10.21883/FTT.2021.09.51279.080

\section{1. Введение}

Значение удельной (на единицу площади) поверхностной энергии $(\sigma)$ кристалла является одним из важнейших параметров, определяющих его прочностные и адгезионные свойства. Поэтому прогнозированию значения $\sigma$ уделяется большое внимание. На сегодняшний день предложено несколько методов расчета величины $\sigma$ для кристалла простого (однокомпонентного) вещества (см., например, [1-15]). Но большинство из этих методов (например, [1,2,5,6,8,9,13,14]) работоспособны только при нулевой температуре $(T=0 \mathrm{~K})$ и нулевом давлении $(P=0)$. Поэтому весьма актуальным является вопрос о зависимости функции $\sigma$ как от температуры $T$, так и от давления $P$, при которых находится кристалл.

В работах $[3,4,7,10,11,12,15]$ были предложены различные методы расчета производной функции $\sigma$ по темпеpaтуре: $\sigma^{\prime}(T)=(\partial \sigma / \partial T)$. Но, ввиду отсутствия в этих работах уравнения состояния для изучаемых веществ, осталось неясным - является ли предложенное в этих работах выражение для $\sigma^{\prime}(T)$ изохорной $\left(\sigma^{\prime}(T)_{v}\right)$ или изобарной $\left(\sigma^{\prime}(T)_{P}\right)$ производной?

Что касается зависимости поверхностной энергии от давления, то выражения для расчета функции $\sigma^{\prime}(P)=(\partial \sigma / \partial P)_{T}$ в литературе пока нет, и поэтому оценок этой величины никто не проводил. Проблема здесь связана с тем, что в теоретических моделях, в рамках которых рассчитывалась функция $\sigma$, уравнение состояния кристалла с учетом поверхности получено не было. Между тем зависимость $\sigma(P)$ необходима при изучении как возникновения трещины при барическом воздействии на макрокристалл, так и для получения уравнения состояния нанокристалла.

В связи с этим, в настоящей работе предложен аналитический метод, позволяющий на основе парного потенциала межатомного взаимодействия, рассчитать, как уравнение состояния, так и функцию $\sigma(P, T)$ при произвольных $P-T$-условиях. Метод позволяет вычислять производные функции $\sigma$ по температуре, как при изохорическом, так и при изобарическом процессе. Метод также позволяет изучить изменение функции $\sigma$ при изотермической вариации как плотности, так и давления. Метод был апробирован для 15 макрокристаллов и показал хорошее согласие с экспериментальными данными. Показано, что метод применим как для макро-, так и для нанокристалла с заданным числом атомов $(N)$ и с определенной формой поверхности.

\section{2. Метод расчета поверхностных Свойств}

Создание аналитической модели поверхности очень сложная и до сих пор не решенная задача. Сложность 
в том, что на поверхности имеется большой градиент плотности, в результате чего атомы на поверхности находятся в динамическом равновесии с обеими фазами, которые и образуют поверхность. Более того, если считать вещество в поверхностном слое как новую фазу (отличную от „объемной“ $)$, то возникают вопросы, как о толщине поверхностного слоя, так и об изменении термодинамических свойств вещества в этом слое при переходе от одной фазы в другую. Чтобы обойти эти проблемы Дж.В. Гиббс (J.W. Gibbs) вместо реальной межфазной границы ввел „разделяющую поверхность“ („dividing surface“ ) [16, гл. 15, стр. 563; 17, гл. 19, стр. 822], к которой относятся поверхностные характеристики. Если реальная поверхность представляет собой переходную зону и имеет некоторую толщину, то разделяющая поверхность это гладкая геометрическая поверхность, которая толщины не имеет. Такую виртуальную поверхность называют поверхностью Гиббса и все рассчитанные для такой модели поверхностные характеристики относятся к гладкой геометрической поверхности не имеющей толщины. Мы будем полагать, что наша модель ограничена поверхностью Гиббса.

Рассмотрим нанокристалл из $N$ одинаковых атомов, ограниченный поверхностью, которая имеет определенную форму и площадь. Изменение свободной энергии Гельмгольца $\left(F_{H}\right)$ такой системы при вариации температуры, объема $(V)$, числа атомов и площади поверхности $(\Sigma)$ обычно представляют в виде $[16$, гл. 2 , стр. 66,$562 ; 17$, гл. 6 , стр. 263$]$ :

$$
\begin{aligned}
d F_{H}= & \left(\frac{\partial F_{H}}{\partial T}\right)_{N, V, \Sigma} d T+\left(\frac{\partial F_{H}}{\partial V}\right)_{N, T, \Sigma} d V \\
& +\left(\frac{\partial F_{H}}{\partial N}\right)_{T, V, \Sigma} d N+\left(\frac{\partial F_{H}}{\partial \Sigma}\right)_{N, V, T} d \Sigma \\
= & -S d T-P d V+\mu_{g} d N+\sigma d \Sigma,
\end{aligned}
$$

где $S$ - энтропия, $P$ - давление, $\mu_{g}-$ химический потенциал и $\sigma$ - удельная (на единицу площади) поверхностная свободная энергия.

Если число атомов в нанокристалле не изменяется, то выражение для $d F_{H}$ можно преобразовать к виду

$$
d f_{H}=d\left(\frac{F_{H}}{N}\right)=-s d T-P d v+\sigma d\left(\frac{\Sigma}{N}\right),
$$

где $s=S / N$ и $v=V / N-$ удельные (на атом) значения энтропии и объема наносистемы.

Из формулы (2) удельная поверхностная энергия нанокристалла равна

$$
\sigma(T, v, N)=\left(\frac{\partial f_{H}}{\partial(\Sigma / N)}\right)_{T, v, N} .
$$

При этом, изменение удельной поверхности $\Sigma / N$ должно происходить обратимым путем, т. е. без необратимого разрушения системы, т.е. без нарушения аксиом равновесной и обратимой термодинамики.
Но при $N=$ const нельзя изоморфно изменить площадь поверхности, не изменив при этом объем, так как при постоянной форме нанокристалла они связаны соотношением: $\Sigma \sim V^{2 / 3}$. Поэтому, как это было указано в $[18,19]$, при $\Sigma / N \neq 0$ определить функцию $\sigma$ можно только путем изохорно-изотермической обратимой деформации формы наносистемы, т.е. из выражения

$$
\begin{aligned}
\sigma(T, v, N, f) & =\left(\frac{\partial f_{H}}{\partial(\Sigma / N)}\right)_{T, N, v} \\
& =\left(\frac{\partial f_{H}}{\partial f}\right)_{T, N, v} /\left(\frac{\partial(\Sigma / N)}{\partial f}\right)_{T, N, v},
\end{aligned}
$$

где $f$ - параметр, определяющий форму системы с конечным значением числа атомов $N$, и которая ограничена поверхностью площадью $\Sigma$.

Из (2) видно, что давление в наносистеме должно вычисляться по формуле

$$
P(T, v, N)=-\left(\frac{\partial f_{H}}{\partial v}\right)_{T, N, \Sigma} .
$$

Но при постоянных значениях $T, N$ и $\Sigma$ невозможно изменить удельный объем наносистемы. Поэтому, для того чтобы обойти данную неопределенность, необходимо принять, что поверхность системы является геометрической поверхностью, не имеющей объема, т. е. поверхностью Гиббса. Это допущение позволяет представить свободную энергию в виде суммы объемного и поверхностного вкладов [16, гл. 15, стр. 563]:

$$
F_{H}(T, v, N, f)=F_{H \text { in }}(T, v)+\sigma(T, c, N, f) \Sigma(c, N, f),
$$

где свободная энергия Гельмгольца для объема наносистемы равна

$$
F_{H \text { in }}(T, v)=N \lim _{N \rightarrow \infty}\left[\frac{F_{H}(T, v, N, f)}{N}\right]_{\nu=\mathrm{const}},
$$

$c=\left(6 k_{p} v / \pi\right)^{1 / 3}$ - среднее (по объему наносистемы) расстояние между центрами ближайших атомов, $k_{p}-$ коэффициентом упаковки структуры из $N$ атомов.

Таким образом, используя (5), определим давление в наносистеме выражением следующего вида:

$$
\begin{aligned}
P(T, v, N, f) & =-\left(\frac{\partial f_{H}}{\partial v}\right)_{T, N} \\
& =P_{i n}(T, v)-P_{s f}(T, c, N, f) .
\end{aligned}
$$

Здесь $P_{\text {in }}$ - это „объемное“ давление т.е. давление, определяемое без учета поверхностного члена в (2) и в (5):

$$
P_{i n}(T, v)=-\lim _{N \rightarrow \infty}\left[\frac{\partial f_{H i n}}{\partial v}\right]_{T, N} .
$$

Функция $P_{s f}$ - это поверхностное давление, которое равно $[19,20]$ :

$$
P_{s f}(T, c, N, f)=\left[\frac{\partial(\sigma \Sigma / N)}{\partial v}\right]_{T, N}=P_{l s}\left(1-\Delta_{p}\right)
$$


Первый сомножитель в (8) это давление Лапласа, которое определяется изменением площади поверхности с изменением объема для наносистемы в вакууме

$$
\begin{aligned}
P_{l s}(T, v, N, f) & =\sigma\left[\frac{\partial(\Sigma / N)}{\partial v}\right]_{T, N} \\
& =\sigma\left(\frac{\Sigma / N}{v}\right)\left[\frac{\partial \ln (\Sigma / N)}{\partial \ln (v)}\right]_{T, N} .
\end{aligned}
$$

Выражение для функции $\Delta_{p}$ из формулы (8) имеет вид

$$
\Delta_{p}=-\left[\frac{\partial \ln (\sigma)}{\partial \ln (\Sigma / N)}\right]_{T, N}=-\left[\frac{\partial \ln (\sigma)}{\partial \ln (\Sigma)}\right]_{T, N} .
$$

Для жидкой фазы выполняется: $(\partial \sigma / \partial \Sigma)_{T, N}=0$. Это обусловлено динамической природой жидкого состояния, где большая доля атомов находится в делокализованном состоянии. Изотермическое растяжение площади ,гиббсовской“ поверхности жидкой фазы вызывает приток к ее поверхности новых атомов из объема. Если приток атомов к поверхности происходит со скоростью, достаточной для того, чтобы поверхностная плотность атомов сохранялась неизменной, то величина $\sigma$ для жидкой фазы не будет меняться с ростом $\Sigma$, и значение $\Delta_{p}$ будет равным нулю. Именно поэтому условие: $\Delta_{P}=0$, как это было показано в [21], можно использовать в качестве критерия „поверхностного“ плавления для системы с геометрической поверхностью Гиббса.

Для твердой фазы считать $\Delta_{p}=0$ нельзя. На это впервые было указано в работе Нагаева [22]. Причем, наличие функции $\Delta_{p}$ в формуле (8) приводит к эффектам, присущим только твердой фазе наносистемы [19,20]:

1) так как $\Delta_{p}>0$, то для нанокристалла всегда выполняется: $P_{s f}<P_{l s}$;

2) если $\Delta_{p}>1$, то поверхностное давление становится растягивающим: $P_{s f}<0$;

3) при плавлении нанокристалла поверхностное давление резко возрастает из-за исчезновения функции $\Delta_{p}$ в $(8)$.

Если кристаллическая структура (характеризуемая коэффициентом упаковки $k_{p}$ ) и форма поверхности (характеризуемая управляющим формой параметром $f$ ) не изменяются при изотермической вариации удельного объема, то функции $P_{l s}$ и $\Delta_{p}$ из $(9)$ и $(10)$ примут вид

$$
\begin{gathered}
P_{l s}=\sigma\left(\frac{\Sigma / N}{v}\right)\left[\frac{\partial \ln (\Sigma / N)}{\partial \ln (v)}\right]_{T, N, k_{p}, f} \\
=\frac{2}{3} \sigma\left(\frac{\Sigma / N}{v}\right), \\
\Delta_{p}=-\left[\frac{\partial \ln (\sigma)}{\partial \ln (\Sigma)}\right]_{T, N}=-\frac{1}{2}\left[\frac{\partial \ln (\sigma)}{\partial \ln (c)}\right]_{T, N, k_{p}, f},
\end{gathered}
$$

Таким образом, для дальнейших расчетов необходимо, используя формулу (3), определить функцию
$\sigma(T, c, N, f)$. Для этого необходимо принять некую геометрическую модель нанокристалла с варьируемой формой поверхности.

\section{3. Геометрическая модель нанокристалла}

Как и в работах $[18-19,23,24]$ положим, что нанокристалл со свободной поверхностью Гиббса имеет вид прямоугольного параллелепипеда с квадратным основанием, ограненный гранями типа (100). Если в решетке нанокристалла простого вещества содержится $N_{v}$ вакансий, однородно распределенных по объему, то первое координационное число (т. е. число ближайших атомов) для атома в объеме нанокристалла равно $[19,23,24]$ :

$$
k_{n}(\infty)=\frac{k_{n}^{o}(\infty) N}{N+N_{v}}=k_{n}^{o}(\infty)\left(1-\phi_{v}\right)
$$

где $\phi_{v}=N_{v} /\left(N_{v}+N\right)$ - вероятность образования вакансии в решетке простого вещества, $k_{n}^{0}(\infty)$ - число ближайших к данному атому ячеек (как занятых, так и вакантных), т. е. это первое координационное число в объеме нанокристалла при $N_{v}=0$.

Величина $f=N_{p s} / N_{p o}=N_{p s}^{o} / N_{p o}^{o}$ - это параметр формы, который определяется отношением числа $N_{p s}^{o}$ атомов (или $N_{p s}=N_{p s}^{o} /\left(1-\phi_{v}\right)^{1 / 3}$ ячеек) на боковом ребре к числу $N_{p o}^{o}$ атомов (или $N_{p o}=N_{p o}^{o} /\left(1-\phi_{\nu}\right)^{1 / 3}$ ячеек) на ребре основания. Очевидно, что для стержневидной формы $f>1$, для куба $f=1$, для пластинчатой формы $f<1$.

Число ячеек и число атомов в нанокристалле определенной формы равно

$$
N+N_{v}=f \frac{N_{p o}^{3}}{\alpha}=f \frac{\left(N_{p o}^{o}\right)^{3}}{\alpha\left(1-\phi_{v}\right)}, \quad N=f \frac{\left(N_{p o}^{o}\right)^{3}}{\alpha},
$$

где $\alpha=\pi /\left(6 k_{p}\right)$ - параметр структуры.

Число атомов и параметр формы в этой модели могут изменяться в пределах

$$
\begin{gathered}
\operatorname{INT}\left[2^{3} / \alpha\right] \leq N \leq \infty \\
\frac{2}{\operatorname{INT}\left[\left(N+N_{v}\right) \alpha / 2\right]^{1 / 2}} \leq f \leq \frac{\operatorname{INT}\left[\left(N+N_{v}\right) \alpha / 4\right]}{2} .
\end{gathered}
$$

Здесь в выражении для $f$ представлены выражения для двух предельных форм модели: левая величина относится к пластине, а правая - к стержню биатомной толщины. Функция INT $[x]$ округляет величину $x$ до целого значения, ибо число атомов или число ячеек по определению являются целыми числами.

Ограничение системы поверхностью приведет к обрыву связей на границе. Поэтому, если использовано приближение „взаимодействия только ближайших соседей“, то вместо первого координационного числа $\left(k_{n}\right)$ необходимо брать функцию $\left\langle k_{n}\right\rangle$, которая есть среднее (по всей 
наносистеме) значение первого координационного числа. Очевидно, что функция $\left\langle k_{n}\right\rangle$ зависит как от размера, так и от формы наносистемы. При этом структуру наносистемы полагаем неизменной: $k_{p}=$ const. Данная модель нанокристалла в виде прямоугольного параллелепипеда (Rectangular Parallelepiped), форму которого можно варьировать с помощью параметра формы $f$, была названа в [18-21] RP-моделью. Аналогичная модель из $N+N_{v}$-ячеек была названа в $[23,24] \mathrm{RP}(\mathrm{vac})$-моделью.

В рамках $\mathrm{RP}(\mathrm{vac})$-модели зависимость нормированного среднего значения первого координационного числа от $N+N_{v}$ и $f$ описывается выражением [23]:

$$
\begin{gathered}
k_{n}^{*}=\frac{\left\langle k_{n}\left(N+N_{v}, f\right)\right\rangle}{k_{n}(\infty)}=1-Z_{s}(f)\left(\frac{\alpha^{2}}{N+N_{v}}\right)^{1 / 3} \\
=1-Z_{s}(f)\left(\frac{\alpha^{2}}{N}\left(1-\phi_{v}\right)\right)^{1 / 3}, \\
Z_{s}(f)=\frac{1+2 f}{3 f^{2 / 3}},
\end{gathered}
$$

где $k_{n}(\infty)=k_{n}^{o}(\infty)\left(1-\phi_{v}\right)$ - координационное число для макрокристалла с учетом вакансий.

Входящая в (15) функция формы $Z_{s}(f)$ достигает минимума равного единице при $f=1$, т. е. для системы в форме куба. Для пластинчатых $(f<1)$ или стержневидных $(f>1)$ форм значение функции формы больше единицы: $Z_{s}(f \neq 1)>1$. Поэтому функция $k_{n}(f)^{*}$ при любом значении $N$ атомов (или $N+N_{v}$ ячеек) имеет максимум при $f=1$, т.е. для наиболее энергетически оптимальной - кубической формы прямоугольного параллелепипеда.

Объем и площадь поверхности для $\mathrm{RP}(\mathrm{vac})$-модели равны

$$
\begin{gathered}
V=\left(N_{p o}^{o}\right)^{3} f c^{3} /\left(1-\phi_{v}\right)=\left(N+N_{v}\right) \alpha c^{3}=\left(N_{p o}^{o}\right)^{3} f c_{o}^{3} \\
\Sigma \\
=6 c^{2} \alpha_{s}\left[\left(N+N_{v}\right) \alpha\right]^{2 / 3} Z_{S}(f) \\
=6 c_{o}^{2} \alpha_{s}(N \alpha)^{2 / 3} Z_{s}(f)
\end{gathered}
$$

где $\alpha_{s}-$ коэффициент, учитывающий плотность упаковки атомов на грани (т.е. в поверхностном слое) нанокристалла: $\alpha_{s} \cong \alpha^{2 / 3} ; \quad c_{o}=\left[6 k_{p} V /(\pi N)\right]^{1 / 3}$ $=c /\left(1-\phi_{v}\right)^{1 / 3}-$ среднее (по объему наносистемы) расстояние между центрами ближайших атомов с учетом вакансий, $c=\left\{6 k_{p} V /\left[\pi\left(N+N_{v}\right)\right]\right\}^{1 / 3}=c_{o}(1-\phi)^{1 / 3}-$ расстояние между центрами ближайших ячеек (как занятых, так и вакантных).

Легко видеть, что объем нанокристалла зависит от формы системы только через зависимость $c(N, f)$.

Кубическая форма может реализовываться только при определенном числе ячеек, из которого можно построить куб

$$
\left(N+N_{\nu}\right)_{\mathrm{cub}}=\left(N_{p o}^{o}\right)^{3} /\left[\alpha\left(1-\phi_{v}\right)\right], \text { где } N_{p o}^{o}=2,3,4, \ldots
$$

При „некубичном“ значении числа ячеек: $N+N_{v}$ $\neq\left(N+N_{v}\right)_{\text {cub }}$, параллелепипед может иметь либо пластинчатую, либо стержневидную форму, причем выполняется неравенство:

$$
k_{n}\left(\left(N+N_{v}\right)_{\mathrm{cub}} \pm 1\right)^{*}<k_{n}\left(N+N_{v}\right)_{\mathrm{cub}}^{*} .
$$

Таким образом, изоморфная (т.е. рассчитанная при $f=\mathrm{const})$ зависимость $k_{n}\left(N+N_{v}\right)$ монотонно уменьшается при $N+N_{v} \rightarrow N_{\min }=\operatorname{INT}\left[2^{3} / \alpha\right]$. Но общая зависимость $k_{n}\left(N+N_{v}\right)$ имеет осциллирующий вид с максимумами в точках $k_{n}\left(N+N_{v}\right)_{\text {cub }}$, соответствующих нанокристаллам с кубической формой, и с минимумами при таких значениях $N+N_{v} \neq\left(N+N_{v}\right)_{\text {cub }}$, из которых можно построить только бездефектный стержень биатомной толщины. А так как многие свойства нанокристалла определяются именно значением $k_{n}(N)$, то зависимость этих свойств от $N$ также будет иметь осциллирующий вид. Поэтому изоморфная производная $\left(\partial k_{n} / \partial N\right)_{f}$ не будет иметь никаких особенностей, чего нельзя сказать о неизоморфной производной $\left(\partial k_{n} / \partial N\right)_{x \neq f}$.

$\mathrm{B}$ рамках $\mathrm{RP}(\mathrm{vac})$-модели удельная поверхностная энергия грани (100) и давление Лапласа определяются выражениями вида

$$
\begin{gathered}
\sigma=\left(\frac{\partial f_{H}}{\partial k_{n}^{*}}\right)_{T, N, c}\left(\frac{\partial k_{n}^{*}}{\partial Z_{S}(f)}\right)_{N, k_{p}} /\left(\frac{\partial(\Sigma / N)}{\partial Z_{s}(f)}\right)_{N, c, k_{p}} \\
=\frac{-\left(1-\phi_{v}\right)}{6 c^{2} \alpha_{s}}\left(\frac{\partial f_{H}}{\partial k_{n}^{*}}\right)_{T, N, c}, \\
P_{l s}=\frac{2 \Sigma}{3 V} \sigma=\frac{4 \alpha_{s} Z_{s}(f)}{\left[\alpha\left(N+N_{v}\right)^{1 / 3} c(N, f)\right]} \sigma \\
=4 \alpha_{s} \frac{\left(1-k_{n}^{*}\right)}{\alpha c(N, f)} \sigma .
\end{gathered}
$$

В „термодинамическом пределе“ (т.е. когда $N \rightarrow \infty$ и $V \rightarrow \infty$ при $v=V / N=$ const), согласно (15), имеем: $k_{n}(N \rightarrow \infty)^{*} \rightarrow 1$. При этом функция $\sigma$ стремится к значению $\sigma(N=\infty)$, а функции $P_{l s}$ из $(18)$ и $P_{s f}$ из $(11)$ исчезают.

Таким образом, для вычисления функции $\sigma(T, c, N, f)$ с помощью (17) необходимо определить зависимость свободной энергии Гельмгольца $f_{H}$ от трех аргументов: $T, c$ и $k_{n}^{*}$.

\section{4. Термодинамические функции для RP-модели}

Пусть взаимодействие атомов в нанокристалле простого (однокомпонентного) вещества описывается парным четырехпараметрическим потенциалом МиЛеннард-Джонса, который имеет следующий вид [17]:

$$
\varphi(r)=\frac{D}{(b-a)}\left[a\left(\frac{r_{o}}{r}\right)^{b}-b\left(\frac{r_{o}}{r}\right)^{a}\right],
$$

где $D$ и $r_{o}$ - глубина и координата минимума потенциала, $b>a \geq 1$ - численные параметры. 
Для простоты допустим, что нанокристалл не содержит вакансий и диффундирующих атомов. Тогда, используя для колебательного спектра нанокристалла модель Эйнштейна и приближение „взаимодействия только ближайших соседей“, для удельной свободной энергии Гельмгольца RP-модели можно принять следующее выражение $[17$, гл. 8$]$ :

$$
\begin{aligned}
\frac{f_{H}}{k_{n}(\infty)}= & \left(\frac{k_{n}^{*}}{2}\right) D U(R)+3 \frac{k_{\mathrm{B}} \Theta_{E}}{k_{n}(\infty)} \\
& \times\left\{\frac{1}{2}+\left(\frac{T}{\Theta_{E}}\right) \ln \left[1-\exp \left(-\frac{\Theta_{E}}{T}\right)\right]\right\} .
\end{aligned}
$$

Здесь $k_{\mathrm{B}}-$ постоянная Больцмана, $\Theta_{E}-$ это температура Эйнштейна, которая связана с температурой Дебая соотношением [17, гл. 8; 25, гл. 2, стр. 116]: $\Theta=(4 / 3) \Theta_{E} ; \quad R=r_{o} / c-$ относительная линейная плотность кристалла, $U(R)$ - функция потенциальной энергии, которая, в соответствии с (19), имеет вид

$$
U(R)=\frac{a R^{b}-b R^{a}}{b-a} .
$$

Как показано в [26], температуру Дебая в рамках сделанных приближений можно определить выражением

$$
\Theta\left(k_{n}, c\right)=A_{w}\left(k_{n}, c\right) \xi\left[-1+\left(1+\frac{8 D}{k_{\mathrm{B}} A_{w}\left(k_{n}, c\right) \xi^{2}}\right)^{1 / 2}\right],
$$

где функция $A_{w}\left(k_{n}, c\right)$ возникает из-за учета энергии „нулевых колебаний“ атомов

$$
\begin{gathered}
A_{w}\left(k_{n}, c\right)=K_{R} \frac{5 k_{n} a b(b+1)}{144(b-a)}\left(\frac{r_{o}}{c}\right)^{b+2}, \\
K_{R}=\frac{\hbar^{2}}{k_{\mathrm{B}} r_{o}^{2} m}, \quad \xi=\frac{9}{k_{n}(\infty)},
\end{gathered}
$$

где $\hbar-$ постоянная Планка, $m-$ масса атома.

Из (21) можно найти выражения для первого $(\gamma)$, второго $(q)$ и третьего $(z)$ параметров Грюнайзена. Они имеют следующий вид:

$$
\begin{gathered}
\gamma=-\left(\frac{\partial \ln \Theta}{\partial \ln v}\right)_{T}=\frac{b+2}{6\left(1+X_{w}\right)}, \\
q=\left(\frac{\partial \ln \gamma}{\partial \ln v}\right)_{T}=\gamma \frac{X_{w}\left(1+2 X_{w}\right)}{\left(1+X_{w}\right)}, \\
z=-\left(\frac{\partial \ln q}{\partial \ln v}\right)_{T}=\gamma\left(1+4 X_{w}\right)-2 q \\
=\gamma\left(\frac{1+3 X_{w}}{1+X_{w}}\right)=\frac{(b+2)}{6} \frac{\left(1+3 X_{w}\right)}{\left(1+X_{w}\right)^{2}},
\end{gathered}
$$

где введена функция: $X_{w}=A_{w} \xi / \Theta$, которая определяет роль квантовых эффектов.
Используя (20)-(23), для уравнения состояния и изотермического модуля упругости $\left(B_{T}\right)$ можно получить следующие выражения [23,27]:

$$
\begin{aligned}
P & =-\left(\frac{\partial f_{H}}{\partial v}\right)_{T} \\
& =\left[\frac{k_{n}}{6} D U^{\prime}(R)+3 k_{\mathrm{B}} \Theta_{E} \gamma E_{w}\left(\frac{\Theta_{E}}{T}\right)\right] \frac{1}{v}, \\
B_{T}= & -v\left(\frac{\partial P}{\partial v}\right)_{T}=P+\left[\frac{k_{n}}{18} D U^{\prime \prime}(R)+3 k_{\mathrm{B}} \Theta_{E} \gamma(\gamma-q)\right. \\
& \left.\times E_{w}\left(\frac{\Theta_{E}}{T}\right)-3 N k_{\mathrm{B}} \gamma^{2} T F_{E}\left(\frac{\Theta_{E}}{T}\right)\right] \frac{1}{v},
\end{aligned}
$$

где функции $k_{n}, \Theta_{E}$ и $\gamma$ зависят от $N-f$-параметров нанокристалла с поверхностью Гиббса,

$$
\begin{gathered}
E_{w}(y)=0.5+\frac{1}{[\exp (y)-1]}, \\
F_{E}(y)=\frac{y^{2} \exp (y)}{[\exp (y)-1]^{2}}, \\
U^{\prime}(R)=R\left[\frac{\partial U(R)}{\partial R}\right]=\frac{a b\left(R^{b}-R^{a}\right)}{b-a}, \\
U^{\prime \prime}(R)=R\left[\frac{\partial U^{\prime}(R)}{\partial R}\right]=\frac{a b\left(b R^{b}-a R^{a}\right)}{b-a} .
\end{gathered}
$$

B рамках RP-модели для удельной (на единицу площади) поверхностной энергии грани (100) нанокристалла, ее изохорной и изобарной производных по температуре и функции $\Delta_{p}$ можно получить следующие выражения [23,27]:

$$
\begin{gathered}
\sigma(N, f)=-\frac{k_{n}(\infty) D R^{2}}{12 \alpha^{2 / 3} r_{o}^{2}} L_{E}(N, f), \\
\sigma^{\prime}(T)_{v}=\left(\frac{\partial \sigma}{\partial T}\right)_{c, N, f} \\
=-\frac{3 k_{\mathrm{B}} R^{2} \gamma(N, f)}{2 \alpha^{2 / 3}(b+2) r_{o}^{2} k_{n}(N, f)^{*}} F_{E}\left(\frac{\Theta_{E}}{T}\right), \\
\sigma^{\prime}(T)_{P}=\left(\frac{\partial \sigma}{\partial T}\right)_{P, N, f}=\sigma^{\prime}(T)_{v}+v \alpha_{P}\left(\frac{\partial \sigma}{\partial v}\right)_{T, N, f} \\
\Delta_{p}=-\frac{1}{2}\left[\frac{\partial \ln (\sigma)}{\partial \ln (c)}\right]_{T, N, k_{p}, f}^{\prime}=1+\frac{2}{2 L_{E}(N, f)} \\
\times\left\{U_{v}^{\prime}(R)-9\left[q-\gamma t_{y}\left(\frac{\Theta_{E}}{T}\right)\right] H_{w}(N, T)\right\} .
\end{gathered}
$$

Здесь $C_{v}$ - изохорная теплоемкость, $\alpha_{p}-$ коэффициент теплового расширения. Эти и введенные в (27)-(30) 
функции имеют следующий вид:

$$
\begin{gathered}
L_{E}(N, f)=U(R)+3 H_{w}(N, T), \quad v_{o}=\frac{\pi r_{o}^{3}}{6 k_{p}}, \\
C_{v}=3 N k_{\mathrm{B}} F_{E}\left(\frac{\Theta_{E}}{T}\right), \\
\alpha_{p}=\frac{1}{v}\left(\frac{\partial v}{\partial T}\right)_{P}=\gamma \frac{C_{v}}{V B_{T}}=\frac{\gamma C_{v}}{N B_{T}\left[\pi r_{o}^{3} /\left(6 k_{p}\right)\right]}\left(\frac{v_{o}}{v}\right), \\
H_{w}(N, T)=\frac{6 \gamma(N, f)}{(b+2)}\left[\frac{k_{\mathrm{B}} \Theta_{E}(N, f)}{D k_{n}(N, f)}\right] E_{w}\left(\frac{\Theta_{E}}{T}\right), \\
t_{y}(y)=1-\frac{2 y \exp (y)}{[\exp (2 y)-1]} .
\end{gathered}
$$

Отметим, что поверхностное давление можно также найти и из выражения (24), как разницу между давлениями, рассчитанными для макро- и нанокристалла:

$$
P_{s f}=P(T, v, N=\infty)-P(T, v, N, f) .
$$

Из полученных выражений (27)-(30) можно сделать следующие выводы:

1. Функция $\sigma(P)$ с ростом давления увеличивается до максимума (при $P_{M}(N, f)$ ), после чего она резко уменьшается и при определенном давлении $\left(P_{f}(N, f)\right)$ переходит в отрицательную область. Более подробно это было изучено в [28].

2. При $P<P_{M}$ для веществ, у которых $\sigma_{p}>0$ выполняется: $\sigma^{\prime}(T)_{P}<\sigma^{\prime}(T)_{v}<0$. При $T=0 \mathrm{~K}$ обе функции при любых $N-f$-параметрах достигают максимума: $\sigma^{\prime}(0)_{P}=\sigma^{\prime}(0)_{v}=0[23,28]$. Это согласуется с третьим началом термодинамики в „сильной“ формулировке Планка: при $T \rightarrow 0$ выполняется $s=-\left(\partial f_{H} / \partial T\right)_{v, N}=0$. Таким образом, зависимости $\sigma^{\prime}(T)_{P}$ и $\sigma^{\prime}(T)_{\nu}$ нелинейные, и полагать (как это сделано в $[7,10,11])$ : $\sigma^{\prime}(T)=$ const, не вполне корректно.

3. Функция $\Delta_{p}$ при $P>P_{M}$ переходит в отрицательную область, а при $P_{f}$ функция $\Delta_{p}$ имеет разрыв второго рода. Подробнее это было изучено в [29].

4. Функции $\sigma(N, f), \sigma^{\prime}(T)_{P}$ и $\sigma^{\prime}(T)_{\nu}$ при $P<P_{M}$ убывают с уменьшением $N$ тем заметнее, чем выше температура, или чем заметнее форма нанокристалла отклонена от энергетически оптимальной формы (для RP-модели это куб) $[18,19,23,28]$.

5. При высоких давлениях и низких температурах функция $\sigma(N)$ может возрастать при изоморфно-изобарно-изотермическом уменьшении числа атомов $N[30,31]$.

Таким образом, полученная в рамках RP-модели функция $k_{n}(N, f)$ вместе с формализмом из (20)-(31) позволяют рассчитать зависимость как уравнения состояния, так и всех решеточных и поверхностных свойств от размера и формы нанокристалла при любых (соответствующих твердой фазе) значениях температуры и удельного объема. Обобщение данной модели на случай наличия в системе вакансий и диффундирующих атомов было сделано в [24]. Это позволило в рамках $\mathrm{RP}(\mathrm{vac})$-модели описать всю фазовую диаграмму простого вещества, включая фазовые переходы кристалл-жидкость, жидкость газ и кристалл-газ. В [24] было показано, что при уменьшении числа атомов в системе S-петля фазового перехода кристалл-жидкость на изотерме уравнения состояния уменьшается, а при определенном значении числа атомов $\left(N_{0}\right) \mathrm{S}$-петля кристалл-жидкость исчезает. При этом величина $N_{0}$ увеличивается как с ростом температуры на изотерме уравнения состояния, так и при отклонении формы нано-системы от наиболее энергетически оптимальной (для $\mathrm{RP}(\mathrm{vac})$-модели - это куб). В кластере из $N<N_{0}$ атомов фазовый переход кристалл-жидкость невозможен. Для металлов была получена оценка: $N_{0}=50-300$.

\section{5. Метод определения параметров межатомного потенциала}

Для расчетов по формулам(20)-(31) необходимо определить параметры парного межатомного потенциала Ми-Леннард-Джонса (19). В литературе встречаются высказывания, что парный четырехпараметрический потенциал Ми-Леннард-Джонса (19) дает при расчетах решеточных свойств кристаллов худшие результаты, чем трехпараметрический потенциал Морзе для металлов, либо многочастичные потенциалы, которые включают не менее 10-12 параметров, и которые можно использовать только в численных расчетах. Это мнение возникло из-за трудностей определения самосогласованным образом всех четырех параметров потенциала Ми-Леннард-Джонса, особенно значений степенней $a$ и $b$. Поэтому ранее (лет 60-70 тому назад) в большинстве расчетов брали ничем не обоснованные значения степенных параметров $a=6$ и $b=12$ [17]. Это было обусловлено тем, что с параметрами 6 и 12 в выражениях для $U(R), U^{\prime}(R)$ и $U^{\prime \prime}(R)$ в (20), (24) и (25) получаются квадратные уравнения, с которым было легче работать. Потенциал 6-12 использовался Дж.Э. Леннард-Джонсом с соавторами (J.E. LennardJones et al.) в ,докомпьютерную эру“ (т.е. в 30-40-х годах ХХв.) для описания свойств инертных газов [17]. По этой же причине потенциал 6-12 (названный в литературе потенциалом Леннард-Джонса) был применен для расчета свойств твердой фазы металлов и диэлектриков. Это привело к плохим результатам, на основе которых и сложилось неправильное мнение о неприменимости потенциала Ми-Леннард-Джонса общего вида (19), т.е. со степенями $a-b$, для описания свойств металлов. Отметим, что потенциал Морзе дает для металлов более лучшие результаты чем потенциал Леннард-Джонса 6-12. Это было обусловлено тем, что в „докомпьютерную эру“ корректно определить три подгоночных параметра было намного легче, чем четыре. Но трехпараметрический потенциал Морзе является частным случаем четырехпарамет- 
рического потенциала Ми-Леннард-Джонса (19) при $b=2 a[17$, Т. 1, стр. 282].

Для определения параметров $r_{o}, D, b$ и $a$ в [32] была предложена система 4-х уравнений, в которые входили измеренные при $T=0 \mathrm{~K}$ и $P=0$ значения молярного объема $\left(V_{00}\right)$, молярной энергии сублимации $\left(L_{00}\right)$, температуры Дебая $\left(\Theta_{00}\right)$ и первого параметра Грюнайзена $\left(\gamma_{00}\right)$. Это позволило в [32] рассчитать параметры потенциала (19) для многих кристаллов: для инертных газов, для металлов и для молекулярных кристаллов.

Но предложенный в [32] метод предполагает, что значения $L_{00}, \Theta_{00}$ и $\gamma_{00}$ определены в экспериментах при $T=0 \mathrm{~K}$ и $P=0$ с высокой точностью. К сожалению, этого не получается, причем наименее точно измеряется величина $\gamma_{00}$. Именно поэтому в [32] значения $D, b$ и $a$ были рассчитаны при фиксированных значениях $r_{o}, L_{00}$ и $\Theta_{00}$ и различных величинах $\gamma_{00}$, которые известны из литературы. При этом возникала неопределенность как в выборе величины $\gamma_{00}$, так и в полученных при таком расчете наборах параметров $b$ и $a$. Это привело к тому, что в работе [33] был предложен метод, в котором параметры $b$ и $a$ определялись при фиксированных значениях $r_{o}$ и молярной энергии сублимации $L_{00}$ и при изменении значений $\Theta_{00}$ и $\gamma_{00}$. Варьируя в широких диапазонах значения $\Theta_{00}$ и $\gamma_{00}: 100<\Theta_{00}<600$ и $1.1<\gamma_{00}<4.1$, из рассчитанных по методу из [32] наборов параметров $D, b$ и $a$ отбираются такие, которые при расчете уравнения состояния: $P\left(T=300 \mathrm{~K}, v / v_{o}=0.8\right)$, модуля упругости: $B_{T}(P=0, T=300 \mathrm{~K})$ и коэффициента теплового расширения: $\alpha_{p}(P=0, T=300 \mathrm{~K})$, дают величины, входящие в интервал допустимых значений.

Данным методом в $[19,33]$ были рассчитаны параметры потенциала (19) для $\alpha$-Fе с объемно-центрированной кубической (ОЦК) структурой, для Аи с гранецентрированной кубической (ГЦК) структурой, и для ряда тугоплавких ОЦК-металлов (Nb, Ta, Mo, W). При этом величину $r_{o}$ брали из работы [34], а значение $L_{00}$ либо из [34], либо (для Та и Мо) из [35]. Однако в работе [36] были получены новые, более корректные значения как для молярной энергии сублимации $L_{00}$, так и для расстояния между центрами ближайших атомов в решетке при $T=0 \mathrm{~K}$ и $P=0$. Поэтому в настоящей работе были определены параметры потенциала (19) как из значений $r_{o}$ и $L_{00}$ представленных в работах $[34,35]$, так и из данных, представленных в [36]. Кроме этого, были рассчитаны параметры межатомного потенциала (19) для ряда других металлов: $\mathrm{Cu}, \mathrm{Ag}, \mathrm{Al}, \mathrm{Rh}$, $\mathrm{Pd}, \mathrm{Pt}, \mathrm{V}, \mathrm{Cr}$.

В табл. 1 и 2 представлены рассчитанные параметры межатомного потенциала (19) и свойства, которые получаются при расчетах с использованием указанных параметров для ГЦК- и ОЦК-металлов. Для всех металлов кроме ОЦК-Сr расчеты выполнены при $P=0$ и $T=300 \mathrm{~K}$. Хром при $T<T_{N}$ является антиферромагнетиком, а при $T>T_{N}$ он переходит в парамагнитное состояние. Так как при $T_{N}(P=0)=312 \mathrm{~K}$ (точка
Нееля) в хроме происходит фазовый переход II рода, то для хрома определение параметров межатомного потенциала (19) проведено при $P=0$ и $T=200 \mathrm{~K}$. Расчеты были выполнены для двух наборов значений энергии сублимации $L_{00}$ и кратчайшего межатомного расстояния $r_{o}-$ из $[34,35]$ и из [36]. Как видно из табл. 1 и 2 , полученные нами величины $P\left(300 \mathrm{~K}, v / v_{o}=0.8\right)$ получились несколько выше экспериментальных данных. Это указывает на то, что рассчитанные параметры потенциала работоспособны в области упругих деформаций, т.е. при $v / v_{o} \geq 0.9$. Но рассчитанные предлагаемым методам потенциальные параметры дают в комплексе лучшее согласие с набором экспериментальных данных, чем параметры потенциала (19) из [32-34].

Помимо определения параметров потенциала (19) данный метод расчета позволяет определить более достоверные значения для $\Theta, \gamma$ и $L_{00}$. Как было указано в $[26,55]$ современные экспериментальные методы не позволяют измерить данные параметры с необходимой точностью (на это указывает разброс экспериментальных данных в табл. 1 и 2). Поэтому использование данного метода весьма актуально.

\section{6. Результаты расчета поверхностных свойств макрокристалла}

В табл. 3 и 4 представлены рассчитанные значения: $v / v_{o}, \quad \sigma \quad\left(\right.$ в $\left.10^{-3} \mathrm{~J} / \mathrm{m}^{2}\right), \quad \sigma^{\prime}(T)_{v} \quad$ и $\quad \sigma^{\prime}(T)_{P}$ (в $\left.10^{-6} \mathrm{~J} /\left(\mathrm{m}^{2} \cdot \mathrm{K}\right)\right), \sigma^{\prime}(P)_{T}=(\partial \sigma / \partial P)_{T}$ - изотермическая производная $\sigma$ по $P\left(\right.$ в $\left.10^{-3} \mathrm{~J} /\left(\mathrm{m}^{2} \cdot \mathrm{GPa}\right)\right)$ и функции $\Delta_{p}$ из (30). Значение $\sigma^{\prime}(P)_{T}$ рассчитывалось путем численного дифференцирования функции $\sigma$ по давлению вдоль изотермы. Данные значения получены при использовании лучших значений параметров потенциала (19) из табл. 1 и 2. В первом столбце табл. 3 и 4 указан источник [34 или 36] откуда были взяты значения $r_{o}$ и $L_{00}$, на основании которых были определены параметры межатомного потенциала.

Для проверки метода расчеты были проведены для макрокристаллов $(N=\infty)$ при $P=0$ и разных значениях температуры. Для каждого металла в первой строке представлены расчеты при $T=300 \mathrm{~K}$ (для $\mathrm{Cr}$ при $200 \mathrm{~K}$ ), во второй при $T=1000 \mathrm{~K}$ (для $\mathrm{Al}$ при температуре его плавления $\left.T_{m}(P=0)=933.52 \mathrm{~K}\right)$. Для металлов, у которых температура плавления $T_{m}(P=0)$ превышает $2600 \mathrm{~K}$ расчеты были выполнены и при $T=2500 \mathrm{~K}$. Эти результаты представлены в табл. 4 в третьей строке. Для Rh-расчеты выполнены при $T=10$, $300,1000,2000$ K. Для каждого металла в нижней строке табл. 3 и 4 представлены известные из литературы экспериментальные и теоретические (в скобках) данные.

Наиболее хорошо поверхностные свойства экспериментально изучены для макрокристаллов ГЦК- $\mathrm{Cu}, \mathrm{Ag}$, $\mathrm{Au}$ и ОЦК-Fе. При этом экспериментальные значения для $\sigma$ и $\sigma^{\prime}(T)_{P}$ оценивались, как правило, при $T \gg \Theta$, а теоретические значения для $\sigma-$ при $T=0 \mathrm{~K}$. Как 
Таблица 1. Энергия сублимации, параметры парного межатомного потенциала (19) и свойства, которые получаются при расчетах с использованием указанных параметров для ГЦК-металлов

\begin{tabular}{|c|c|c|c|c|c|c|c|c|c|c|c|}
\hline $\begin{array}{l}\text { Metal } \\
m[\mathrm{amu}]\end{array}$ & $\begin{array}{c}L_{00} \\
\mathrm{~kJ} / \mathrm{mol}\end{array}$ & $\begin{array}{c}r_{o}, \\
10^{-10} \mathrm{~m}\end{array}$ & $\begin{array}{l}D / k_{B} \\
\quad \mathrm{~K}\end{array}$ & $b$ & $a$ & $\begin{array}{c}P(0.8) \\
\mathrm{GPa}\end{array}$ & $\begin{array}{c}\alpha_{p} \\
10^{-6} \mathrm{~K}^{-1}\end{array}$ & $\begin{array}{c}B_{T}=-v(\partial P / \partial v)_{T} \\
\operatorname{GPa}\end{array}$ & $\begin{array}{c}B^{\prime}(P)= \\
\left(\partial B_{T} / \partial P\right)_{T}\end{array}$ & $\stackrel{\Theta_{00}}{\mathrm{~K}}$ & $\gamma_{00}$ \\
\hline \multirow{3}{*}{$\begin{array}{c}\mathrm{Cu} \\
63.546\end{array}$} & $338.07[34]$ & $2.5487[34]$ & 6838.00 & 9.69 & 2.70 & 65.8 & 49.34 & 130.01 & 6.18 & 316.03 & 1.940 \\
\hline & $338.66[36]$ & $2.5440[36]$ & 6849.84 & 9.63 & 2.70 & 65.7 & 49.29 & 130.19 & 6.16 & 316.43 & 1.930 \\
\hline & \multicolumn{5}{|c|}{ Experiments } & $\begin{array}{c}51.6[37] \\
53.374[38]\end{array}$ & $\begin{array}{c}49.5[37] \\
49.19[38] \\
50.1[39]\end{array}$ & $\begin{array}{c}137[37,41] \\
133.5[38] \\
125.3-165[40]\end{array}$ & $5.32[38]$ & $\begin{array}{c}310-344[35] \\
343[41] \\
345.6 \pm 1.0[42]\end{array}$ & $\begin{array}{c}1.7-2.04[35] \\
1.983[37] \\
1.956[38]\end{array}$ \\
\hline \multirow{3}{*}{$\begin{array}{c}\mathrm{Ag} \\
107.868\end{array}$} & $284.09[34]$ & $2.8760[34]$ & 5735.82 & 10.28 & 2.94 & 45.79 & 55.66 & 86.68 & 6.46 & 211.54 & 2.040 \\
\hline & $285.60[36]$ & $2.8770[36]$ & 5766.03 & 10.28 & 2.93 & 45.81 & 55.54 & 86.78 & 6.46 & 211.54 & 2.040 \\
\hline & \multicolumn{5}{|c|}{ Experiments } & $\begin{array}{l}43.459[38] \\
42-45[43] \\
40-44[40]\end{array}$ & $\begin{array}{c}55.82[38] \\
56.7[39]\end{array}$ & $\begin{array}{c}100.00[38] \\
82-111[40] \\
99.32[43]\end{array}$ & $\begin{array}{l}6.15[38] \\
5.89[43]\end{array}$ & $\begin{array}{c}215-231[35] \\
226.6 \pm 1.0[42]\end{array}$ & $\begin{array}{c}2.05-2.8[35] \\
2.350[38] \\
2.05 \pm 0.07[39]\end{array}$ \\
\hline \multirow{2}{*}{$\begin{array}{c}\mathrm{Au} \\
196.967\end{array}$} & $368.19[34]$ & $2.8751[34]$ & 7419.16 & 16.05 & 2.80 & 109.88 & 43.00 & 166.38 & 8.35 & 198.04 & 3.001 \\
\hline & \multicolumn{5}{|c|}{ Experiments } & $\begin{array}{c}71.053[38] \\
65-75[43] \\
72[40]\end{array}$ & $\begin{array}{c}41.47[38] \\
42-42.8[39,44]\end{array}$ & $\begin{array}{c}167.00[38] \\
135.7-186[40]\end{array}$ & $\begin{array}{l}5.90[38] \\
5.31[43] \\
9.57[44]\end{array}$ & $\begin{array}{c}156-178[35] \\
162.4 \pm 2.0[42]\end{array}$ & $\begin{array}{c}2.3-3.05[35] \\
2.888[38] \\
2.92[39]\end{array}$ \\
\hline \multirow{2}{*}{$\begin{array}{c}\mathrm{Al} \\
26.982\end{array}$} & $326.77[34]$ & $2.8541[34]$ & 6620.30 & 11.15 & 1.96 & 39.94 & 69.82 & 72.07 & 6.43 & 355.06 & 2.181 \\
\hline & \multicolumn{5}{|c|}{ Experiments } & $\begin{array}{c}26.6[37] \\
26.665[38]\end{array}$ & $\begin{array}{c}69.30[37] \\
68.51[38] \\
69.9[39]\end{array}$ & $\begin{array}{l}73.15[37] \\
72.80[38]\end{array}$ & $4.51[38]$ & $\begin{array}{c}390-430.6[35] \\
426.7-437.1[42]\end{array}$ & $\begin{array}{c}2.13-2.7[35] \\
2.088[37] \\
2.166[38]\end{array}$ \\
\hline \multirow[t]{2}{*}{$\begin{array}{c}\mathrm{Ni} \\
58.69\end{array}$} & $\begin{array}{l}430.12[34] \\
432.25[36]\end{array}$ & $\begin{array}{l}2.4780[34] \\
2.4840[36]\end{array}$ & $\begin{array}{l}8694.42 \\
8736.92\end{array}$ & $\begin{array}{l}9.33 \\
9.39\end{array}$ & $\begin{array}{l}2.67 \\
2.65\end{array}$ & $\begin{array}{l}84.76 \\
84.61\end{array}$ & $\begin{array}{l}38.10 \\
38.20\end{array}$ & $\begin{array}{l}174.27 \\
173.57\end{array}$ & $\begin{array}{l}6.04 \\
6.05\end{array}$ & $\begin{array}{l}377.37 \\
375.93\end{array}$ & $\begin{array}{l}1.880 \\
1.891\end{array}$ \\
\hline & \multicolumn{5}{|c|}{ Experiments } & $\begin{array}{l}70-76[40] \\
72-85[45] \\
63-75[46]\end{array}$ & $\begin{array}{c}39[39] \\
38-47[46]\end{array}$ & $\begin{array}{c}171-198[40] \\
176.7-192[46]\end{array}$ & $4.3-5.23[46]$ & $\begin{array}{c}345-476[35] \\
471.2-491.9[42]\end{array}$ & $\begin{array}{c}1.6-1.91[35] \\
1.6[39] \\
1.83-1.89[45]\end{array}$ \\
\hline $\begin{array}{c}\mathrm{Rh} \\
102.906\end{array}$ & \multicolumn{5}{|c|}{ Experiments } & $\begin{array}{l}90-110[40] \\
60-110[47]\end{array}$ & $\begin{array}{c}25.5[39] \\
25.44[48]\end{array}$ & $\begin{array}{l}122-388[40] \\
170-285[47] \\
261-310[48]\end{array}$ & $2.9-4.6[48]$ & $\begin{array}{l}340-480[35] \\
480[47]\end{array}$ & $\begin{array}{c}2.26-2.34[35] \\
0.8-2.8[47]\end{array}$ \\
\hline \multirow[t]{2}{*}{$\begin{array}{c}P d \\
106.42\end{array}$} & $\begin{array}{l}379.91[34] \\
379.19[36]\end{array}$ & $\begin{array}{l}2.7432[34] \\
2.7450[36]\end{array}$ & $\begin{array}{l}7671.24 \\
7656.80\end{array}$ & $\begin{array}{l}10.04 \\
10.04\end{array}$ & $\begin{array}{l}3.43 \\
3.43\end{array}$ & $\begin{array}{l}79.81 \\
79.51\end{array}$ & $\begin{array}{l}34.19 \\
34.26\end{array}$ & $\begin{array}{l}157.10 \\
156.50\end{array}$ & $\begin{array}{l}6.53 \\
6.53\end{array}$ & $\begin{array}{l}290.94 \\
290.48\end{array}$ & $\begin{array}{l}2.000 \\
2.000\end{array}$ \\
\hline & \multicolumn{5}{|c|}{ Experiments } & $62-76[40]$ & $35.25[39]$ & $\begin{array}{c}155-208[40] \\
183[43]\end{array}$ & $5.28[43]$ & $\begin{array}{c}265-296[35] \\
270[42]\end{array}$ & $\begin{array}{l}2.18-2.3[35] \\
2.3 \pm 0.2[39]\end{array}$ \\
\hline \multirow{3}{*}{$\begin{array}{c}\mathrm{Pt} \\
195.08\end{array}$} & $564.84[34]$ & $2.7689[34]$ & 11370.04 & 11.71 & 3.05 & 124.91 & 25.42 & 237.77 & 6.95 & 248.75 & 2.280 \\
\hline & $566.37[36]$ & $2.7660[36]$ & 11400.70 & 11.65 & 3.05 & 124.69 & 25.34 & 237.97 & 6.93 & 249.00 & 2.270 \\
\hline & \multicolumn{5}{|c|}{ Experiments } & $\begin{array}{l}110.566[38] \\
72-108[40]\end{array}$ & $\begin{array}{l}27.18[38] \\
26.97[39]\end{array}$ & $\begin{array}{c}275[38] \\
237-296[40]\end{array}$ & $\begin{array}{l}5.35[38] \\
5.03[43]\end{array}$ & $\begin{array}{c}225-240[35] \\
234.9 \pm 0.4[42]\end{array}$ & $\begin{array}{c}2.55-2.7[35] \\
2.79[38]\end{array}$ \\
\hline
\end{tabular}

видно из табл. 3 и 4 наши расчеты величины $\sigma(100)$ хорошо согласуются с экспериментальными и теоретическими оценками из работ других авторов. Что касается производных функции $\sigma$ по $T$ и $P$, то в литературе имеется очень мало данных для $\sigma^{\prime}(T)_{P}$, а для $\sigma^{\prime}(T)_{\nu}$, $\sigma^{\prime}(P)$ и $\Delta_{p}$ никаких данных (ни теоретических, ни экспериментальных) в литературе нет.

В работах $[4,6,7,56-57]$ точность экспериментального определения величины $\sigma(100)$ для металлов оценивают в пределах $\pm(100-200) \cdot 10^{-3} \mathrm{~J} / \mathrm{m}^{2}$. Численные методы расчета $\sigma(100)$, согласно $[6,8,10,12-14,60]$, имеют точность $\pm(180-640) \cdot 10^{-3} \mathrm{~J} / \mathrm{m}^{2}$. В связи с этим, определить значение $\sigma^{\prime}(T)_{P}$, которое по величине порядка $10^{-(4-5)} \mathrm{J} /\left(\mathrm{m}^{2} \cdot \mathrm{K}\right)$, экспериментально, либо путем численного моделирования очень проблематично. Исходя из этого, можно утверждать, что полученные здесь значения $\sigma^{\prime}(T)_{P}$ являются более корректными, чем известные из литературы экспериментальные оценки. 
Таблица 2. Энергия сублимации, параметры парного межатомного потенциала (19) и свойства, которые получаются при расчетах с использованием указанных параметров для ОЦК-металлов

\begin{tabular}{|c|c|c|c|c|c|c|c|c|c|c|c|}
\hline $\begin{array}{c}\text { Metal } \\
m[\mathrm{amu}]\end{array}$ & $\begin{array}{c}L_{00} \\
\mathrm{~kJ} / \mathrm{mol}\end{array}$ & $\begin{array}{c}r_{o} \\
10^{-10} \mathrm{~m}\end{array}$ & $\begin{array}{c}D / k_{B} \\
\mathrm{~K}\end{array}$ & $b$ & $a$ & $\begin{array}{c}P(0.8) \\
\mathrm{GPa}\end{array}$ & $\begin{array}{c}\alpha_{p} \\
10^{-6} \mathrm{~K}^{-1}\end{array}$ & $\begin{array}{c}B_{T}=-v(\partial P / \partial v)_{T} \\
\operatorname{GPa}\end{array}$ & $\begin{array}{c}B^{\prime}(P)= \\
\left(\partial B_{T} / \partial P\right)_{T}\end{array}$ & $\stackrel{\Theta_{00}}{\mathrm{~K}}$ & $\gamma_{00}$ \\
\hline \multirow{2}{*}{$\begin{array}{c}\alpha-\mathrm{Fe} \\
55.847\end{array}$} & $413.80[34]$ & $2.4775[34]$ & 12561.53 & 8.37 & 3.09 & 75.93 & 34.12 & 161.29 & 5.85 & 415.17 & 1.720 \\
\hline & \multicolumn{5}{|c|}{ Experiments } & $50-55[45]$ & $33-38[39,49]$ & $156-171[49,50]$ & $4-6[49,50]$ & $\begin{array}{l}420-478[25,35] \\
466.7-486.9[42]\end{array}$ & $1.4-1.8[35]$ \\
\hline \multirow{2}{*}{$\begin{array}{c}\mathrm{V} \\
50.942\end{array}$} & $515.23[36]$ & $2.6060[36]$ & 15622.48 & 7.52 & 3.08 & 69.92 & 27.35 & 156.24 & 5.56 & 455.97 & 1.580 \\
\hline & \multicolumn{5}{|c|}{ Experiments } & $50-62[45]$ & $\begin{array}{c}23.52[39] \\
24-30[51]\end{array}$ & $\begin{array}{c}162[43], 157-198[52] \\
151-157[53]\end{array}$ & $\begin{array}{l}3.5[43], 3-4[52] \\
\quad 3.5-4.27[53]\end{array}$ & $\begin{array}{c}273-390[35] \\
345.6 \pm 1.0[42]\end{array}$ & $1.0-1.38[35]$ \\
\hline \multirow{3}{*}{$\begin{array}{c}\mathrm{Nb} \\
92.9064\end{array}$} & $719.65[34]$ & $2.8648[34]$ & 21732.20 & 9.24 & 2.55 & 77.66 & 24.01 & 167.21 & 5.95 & 327.28 & 1.869 \\
\hline & $720.75[36]$ & $2.8540[36]$ & 21765.36 & 9.34 & 2.53 & 79.16 & 24.13 & 169.78 & 5.98 & 327.84 & 1.890 \\
\hline & \multicolumn{5}{|c|}{ Experiments } & $\begin{array}{l}57.087[38] \\
50-60[43]\end{array}$ & $\begin{array}{c}20.8[38] \\
21.3-22.8[39,51]\end{array}$ & $\begin{array}{c}170.5[38], 168.8[43] \\
164-174[52] \\
169-178[53]\end{array}$ & $\begin{array}{c}3.65[38], 3.3[43] \\
3.1-3.85[52] \\
3.6-4.03[53]\end{array}$ & $\begin{array}{c}260-280[35] \\
300[41], 277[42]\end{array}$ & $\begin{array}{c}1.3-1.69[35] \\
1.587[38]\end{array}$ \\
\hline \multirow{3}{*}{$\begin{array}{c}\mathrm{Ta} \\
180.948\end{array}$} & $706.26[34]$ & $2.8648[34]$ & 21318.49 & 7.86 & 3.49 & 86.25 & 18.53 & 192.81 & 5.80 & 289.93 & 1.640 \\
\hline & $782.50[36]$ & $2.8560[36]$ & 23608.03 & 7.74 & 3.16 & 84.58 & 18.58 & 192.30 & 5.65 & 280.47 & 1.621 \\
\hline & \multicolumn{5}{|c|}{ Experiments } & $\begin{array}{l}64.965[38] \\
55-75[43]\end{array}$ & $\begin{array}{c}19.74[38] \\
18-19.8[39,51]\end{array}$ & $\begin{array}{c}191[38], 192[43] \\
194-211[52] \\
194.2-201[53]\end{array}$ & $\begin{array}{c}3.83[38] \\
3.52-3.67[52] \\
3.6-3.8[53]\end{array}$ & $\begin{array}{c}225-264.6[35] \\
160.9[43]\end{array}$ & $1.5-2.19[35]$ \\
\hline \multirow[t]{2}{*}{$\begin{array}{c}\mathrm{Cr} \\
51.996\end{array}$} & $\begin{array}{l}397.48[34] \\
400.41[36]\end{array}$ & $\begin{array}{l}2.4950[34] \\
2.4850[36]\end{array}$ & $\begin{array}{l}12133.31 \\
12222.45\end{array}$ & $\begin{array}{l}8.28 \\
6.40\end{array}$ & $\begin{array}{l}4.78 \\
4.41\end{array}$ & $\begin{array}{c}114.80 \\
76.96\end{array}$ & $\begin{array}{l}15.50 \\
17.48\end{array}$ & $\begin{array}{l}239.03 \\
173.81\end{array}$ & $\begin{array}{l}6.37 \\
5.62\end{array}$ & $\begin{array}{l}638.69 \\
644.14\end{array}$ & $\begin{array}{l}1.701 \\
1.390\end{array}$ \\
\hline & \multicolumn{5}{|c|}{ Experiments } & $70-85[45]$ & $15.54[39]$ & $\begin{array}{c}190.1[41] \\
165-199[53]\end{array}$ & $4.89[53]$ & $\begin{array}{l}460-630[35] \\
\quad 630[41]\end{array}$ & $1.51-1.91[35]$ \\
\hline $\begin{array}{c}\text { Mo } \\
95.94\end{array}$ & $\begin{array}{c}654.867[35] \\
659.96[36]\end{array}$ & $\begin{array}{l}2.7200[32] \\
2.7210[36]\end{array}$ & $\begin{array}{l}19820.02 \\
19972.52\end{array}$ & $\begin{array}{l}8.05 \\
8.05\end{array}$ & $\begin{array}{l}3.96 \\
3.93\end{array}$ & $\begin{array}{l}111.58 \\
111.34\end{array}$ & $\begin{array}{l}16.39 \\
16.41\end{array}$ & $\begin{array}{l}243.32 \\
243.08\end{array}$ & $\begin{array}{l}6.02 \\
6.02\end{array}$ & $\begin{array}{l}454.70 \\
452.91\end{array}$ & $\begin{array}{l}1.670 \\
1.670\end{array}$ \\
\hline \multirow[t]{2}{*}{$\begin{array}{c}\mathrm{W} \\
183.85\end{array}$} & $\begin{array}{l}848.10[34] \\
851.97[36]\end{array}$ & $\begin{array}{l}2.7365[34] \\
2.7380[36]\end{array}$ & $\begin{array}{l}25594.79 \\
25710.94\end{array}$ & $\begin{array}{l}8.82 \\
8.82\end{array}$ & $\begin{array}{l}3.45 \\
3.44\end{array}$ & $\begin{array}{l}136.25 \\
136.20\end{array}$ & $\begin{array}{l}15.03 \\
15.01\end{array}$ & $\begin{array}{l}295.66 \\
295.66\end{array}$ & $\begin{array}{l}6.12 \\
6.10\end{array}$ & $\begin{array}{l}330.35 \\
330.13\end{array}$ & $\begin{array}{l}1.800 \\
1.800\end{array}$ \\
\hline & \multicolumn{5}{|c|}{ Experiments } & $\begin{array}{c}108.518[38] \\
103-120[43]\end{array}$ & $\begin{array}{c}11.94[38] \\
11-15[39,51]\end{array}$ & $\begin{array}{c}308[38], 296[43] \\
323.3[41], \\
296-329[52] \\
306.5-318[53]\end{array}$ & $\begin{array}{c}4.12[38], 4.4[43] \\
3.89-4.3[52] \\
3.9-4.32[53]\end{array}$ & $\begin{array}{c}310-610[35] \\
400[41], 390[42] \\
232[43]\end{array}$ & $\begin{array}{c}1.1-1.76[35] \\
1.404[38]\end{array}$ \\
\hline
\end{tabular}

\section{7. Изменение поверхностных свойств ГЦК-родия при переходе от макро- К нанокристаллу}

Из представленных в табл. 1-4 металлов наименее изучен ГЦК-родий. Для Rh имеем следующие данные:

$$
\begin{gathered}
m(\mathrm{Rh})=102.906 \mathrm{amu}, k_{n}^{o}(\infty)=12, k_{p}=0.7405, \\
\alpha=\pi /\left(6 k_{p}\right)=0.70709, \\
v_{o}=\left[\pi /\left(6 k_{p}\right)\right] r_{o}^{3}=11.478\left[10^{-30} \mathrm{~m}^{3}\right], \\
V_{o}=v_{o} N_{A}=6.9122\left[\mathrm{~cm}^{3} / \mathrm{mol}\right],
\end{gathered}
$$

где $N_{A}$ - число Авогадро.
При $P=0$ и $T=1033 \pm 5 \mathrm{~K}[61]$ в $\mathrm{Rh}$ наблюдается фазовый переход II рода, и Rh имеет температуру плавления равную $T_{m}(\mathrm{Rh})=2236 \pm 3 \mathrm{~K}$ [61]. Поэтому изменения поверхностных свойств ГЦК-Rh были рассчитаны вдоль трех изотерм: $T=10,300,2000 \mathrm{~K}$.

На рис. 1-3 представлены барические зависимости поверхностных свойств для ГЦК-Rh, атомы которого взаимодействуют посредством парного потенциала (19) с параметрами со степенями 3.35-5.34 из табл. 1 и 3. Расчеты были выполнены для макрокристалла $\left(N=\infty, k_{n}^{*}=1\right)$, и для нанокристалла кубической формы $(f=1)$ при $N_{p o}=7$, т.е. состоящего из $N=\operatorname{INT}\left[f N_{p o}^{3} / \alpha\right]=485$ атомов $\left(k_{n}^{*}=0.898987\right.$, т.е. $\left.k_{n}=10.788\right)$. На рис. $1-3$ толстыми сплошными линиями 1, 3, 5 показаны зависимости для макрокристал- 
Таблица 3. Значения поверхностных свойств рассчитанные для макрокристаллов ГЦК-металлов при $P=0$ (Для каждого кристалла в первой строке представлены расчеты при $T=300 \mathrm{~K}$, во второй при $T=1000 \mathrm{~K}$. В нижней строке в скобках представлены известные из литературы экспериментальные и теоретические данные)

\begin{tabular}{|c|c|c|c|c|c|c|c|}
\hline $\begin{array}{l}\left.\text { Metal }\left(T_{m}(P=0) / \mathrm{K}\right)[35]\right) \\
\text { Parameters of potential }(19)\end{array}$ & $\begin{array}{l}T \\
\mathrm{~K}\end{array}$ & $v / v_{o}$ & $\begin{array}{c}\sigma(100), \\
10^{-3} \mathrm{~J} / \mathrm{m}^{2}\end{array}$ & $\begin{array}{c}-\sigma^{\prime}(T)_{v} \\
\mathrm{mkJ} /\left(\mathrm{m}^{2} \mathrm{~K}\right)\end{array}$ & $\begin{array}{c}-\sigma^{\prime}(T)_{P} \\
\mathrm{mkJ} /\left(\mathrm{m}^{2} \mathrm{~K}\right)\end{array}$ & $\begin{array}{c}\sigma^{\prime}(P)_{T} \\
\mathrm{~mJ} /\left(\mathrm{m}^{2} \mathrm{GPa}\right)\end{array}$ & $\Delta_{p}$ \\
\hline \multirow{2}{*}{$\begin{array}{l}\mathrm{Cu}(1357.65) \\
r_{o}=2.544\left[10^{-10} \mathrm{~m}\right] \\
D / k_{B}=6849.84[\mathrm{~K}] \\
b=9.63 ; a=2.70[36]\end{array}$} & $\begin{array}{c}300 \\
1000\end{array}$ & $\begin{array}{l}1.01598 \\
1.05628\end{array}$ & $\begin{array}{l}1800.28 \\
1703.25\end{array}$ & $\begin{array}{l}62.85 \\
64.27\end{array}$ & $\begin{array}{l}125.81 \\
148.96\end{array}$ & $\begin{array}{c}9.81 \\
13.07\end{array}$ & $\begin{array}{l}1.0642 \\
1.2207\end{array}$ \\
\hline & \multicolumn{2}{|c|}{$\begin{array}{l}\text { Experiments } \\
\text { and (theories) }\end{array}$} & $\begin{array}{c}1790-1830[1],(2170)[1], 1830[2,3],(2090-2145)[2] \\
(1683)[3], 1790 \pm 190[8],(1480-2170)[8],(1686)[9] \\
1520 \pm 14[56], 1473[57], 1790-1920[58]\end{array}$ & & $\begin{array}{c}500[57] \\
70-100[58] \\
(204)[59]\end{array}$ & & \\
\hline \multirow{2}{*}{$\begin{array}{l}\operatorname{Ag}(1235.08) \\
r_{o}=2.877\left[10^{-10} \mathrm{~m}\right] \\
D / k_{B}=5766.03[\mathrm{~K}] \\
b=10.28 ; a=2.93[36]\end{array}$} & $\begin{array}{c}300 \\
1000\end{array}$ & $\begin{array}{c}1.01695 \\
1.0627\end{array}$ & $\begin{array}{l}1181.89 \\
1107.10\end{array}$ & $\begin{array}{l}50.59 \\
50.22\end{array}$ & $\begin{array}{c}97.89 \\
115.67\end{array}$ & $\begin{array}{c}9.81 \\
13.88\end{array}$ & $\begin{array}{l}1.0808 \\
1.2793\end{array}$ \\
\hline & \multicolumn{2}{|c|}{$\begin{array}{l}\text { Experiments } \\
\text { and (theories) }\end{array}$} & $\begin{array}{c}1250[1-3],(1200-1400)[\mathbf{1}],(1210-2167)[2] \\
(1175)[3], 1250 \pm 130[8],(810-1270)[8] \\
1205 \pm 26[56], 1155[57]\end{array}$ & & $\begin{array}{c}400[57] \\
(123)[59]\end{array}$ & & \\
\hline \multirow{2}{*}{$\begin{array}{l}\text { Au }(1337.58) \\
r_{o}=2.8751\left[10^{-10} \mathrm{~m}\right] \\
D / k_{B}=7419.16[\mathrm{~K}] \\
b=16.05 ; a=2.80[34]\end{array}$} & $\begin{array}{c}300 \\
1000\end{array}$ & $\begin{array}{l}1.01302 \\
1.04803\end{array}$ & $\begin{array}{l}1531.38 \\
1454.77\end{array}$ & $\begin{array}{l}50.98 \\
50.80\end{array}$ & $\begin{array}{c}98.93 \\
120.53\end{array}$ & $\begin{array}{l}6.70 \\
9.89\end{array}$ & $\begin{array}{l}1.0923 \\
1.3159\end{array}$ \\
\hline & \multicolumn{2}{|c|}{$\begin{array}{l}\text { Experiments } \\
\text { and (theories) }\end{array}$} & $\begin{array}{c}1500 \pm 1510[1],(1630-1800)[1] \\
1500[2,3],(864-1627)[2],(1542)[3], 1510 \pm 160[8] \\
(850-1710)[8], 1410 \pm 37[56], 1363[57]\end{array}$ & & $\begin{array}{l}\mathbf{5 0 0}[57] \\
(\mathbf{9 2})[59]\end{array}$ & & \\
\hline \multirow{2}{*}{$\begin{array}{l}\text { Al }(933.52) \\
r_{o}=2.841\left[10^{-10} \mathrm{~m}\right] \\
D / k_{B}=6620.30[\mathrm{~K}] \\
b=11.15 ; a=1.96[34]\end{array}$} & $\begin{array}{c}300 \\
933.52\end{array}$ & \begin{tabular}{|l|}
1.02303 \\
1.07771
\end{tabular} & $\begin{array}{l}1374.94 \\
1289.32\end{array}$ & $\begin{array}{l}49.01 \\
50.29\end{array}$ & $\begin{array}{l}117.83 \\
150.80\end{array}$ & $\begin{array}{l}13.68 \\
19.81\end{array}$ & $\begin{array}{l}1.0754 \\
1.2413\end{array}$ \\
\hline & \multicolumn{2}{|c|}{$\begin{array}{l}\text { Experiments } \\
\text { and (theories) }\end{array}$} & $\begin{array}{c}1140-1160[1],(1350-1680)[1], 1140 \pm 200[8] \\
(860-1350)[8],(921)[9], 1140 \pm 200[56,57] \\
(1000.6-989)[60]\end{array}$ & & $\begin{array}{l}(\mathbf{1 0 3})[59] \\
(\mathbf{1 0 7})[60]\end{array}$ & & \\
\hline \multirow{2}{*}{$\begin{array}{l}\mathbf{N i}(1728) \\
r_{o}=2.484\left[10^{-10} \mathrm{~m}\right] \\
D / k_{B}=8736.92[\mathrm{~K}] \\
b=9.39 ; a=2.65[36]\end{array}$} & $\begin{array}{c}300 \\
1000\end{array}$ & $\begin{array}{l}1.01302 \\
1.04383\end{array}$ & $\begin{array}{c}241.14 \\
2319.40\end{array}$ & $\begin{array}{l}64.69 \\
67.83\end{array}$ & $\begin{array}{l}129.34 \\
151.47\end{array}$ & $\begin{array}{c}9.75 \\
12.15\end{array}$ & $\begin{array}{l}1.0494 \\
1.1679\end{array}$ \\
\hline & \multicolumn{2}{|c|}{$\begin{array}{l}\text { Experiments } \\
\text { and (theories) }\end{array}$} & $\begin{array}{c}2380-2450[1],(2430-2880)[1], 2450[3],(2538)[3] \\
(2210)[5], 1940 \pm 46[56], 1920[57]\end{array}$ & & $\begin{array}{c}\mathbf{5 0 0}[\mathbf{5 7}] \\
(\mathbf{1 3 2})[\mathbf{5 9}]\end{array}$ & & \\
\hline \multirow[t]{2}{*}{$\begin{array}{l}\mathbf{R h}(2236) \\
r_{o}=2.532\left[10^{-10} \mathrm{~m}\right] \\
D / k_{B}=11214.52[\mathrm{~K}] \\
b=5.34 ; a=3.35[36]\end{array}$} & $\begin{array}{c}10 \\
300 \\
1000 \\
2000\end{array}$ & $\begin{array}{l}1.00409 \\
1.00897 \\
1.02914 \\
1.06158\end{array}$ & $\begin{array}{l}3024.77 \\
3002.98 \\
2916.12 \\
2784.18\end{array}$ & $\begin{array}{l}\sim 10^{-8} \\
62.13 \\
65.89 \\
64.88\end{array}$ & $\begin{array}{l}\sim 10^{-8} \\
115.26 \\
128.12 \\
135.77\end{array}$ & $\begin{array}{l}12.75 \\
13.18 \\
14.65 \\
17.12\end{array}$ & $\begin{array}{l}1.0061 \\
1.0246 \\
1.0837 \\
1.1716\end{array}$ \\
\hline & \multicolumn{2}{|c|}{$\begin{array}{l}\text { Experiments } \\
\text { and (theories) }\end{array}$} & $\begin{array}{l}2660-2700[1],(2800-3150)[1] \\
2700[2,3],(2810-3010)[2],(2600)[3] \\
\quad 2660 \pm 290[8],(2710-3040)[8]\end{array}$ & & & & \\
\hline \multirow{2}{*}{$\begin{array}{l}\text { Pd }(1827) \\
r_{o}=2.7432\left[10^{-10} \mathrm{~m}\right] \\
D / k_{B}=7671.24[\mathrm{~K}] \\
b=10.04 ; a=3.43[34]\end{array}$} & $\begin{array}{c}300 \\
1000\end{array}$ & $\begin{array}{l}1.01102 \\
1.03785\end{array}$ & $\begin{array}{l}1742.17 \\
1669.28\end{array}$ & $\begin{array}{l}54.71 \\
55.99\end{array}$ & $\begin{array}{c}96.79 \\
109.81\end{array}$ & $\begin{array}{l}7.83 \\
9.88\end{array}$ & $\begin{array}{l}1.0596 \\
1.2030\end{array}$ \\
\hline & \multicolumn{2}{|c|}{$\begin{array}{l}\text { Experiments } \\
\text { and (theories) }\end{array}$} & $\begin{array}{c}2000-2050[1],(2150-2330)[1] \\
2050[2,3],(1490-2151)[2],(1690)[3], 2000 \pm 220[8] \\
(1490-2430)[8]\end{array}$ & & & & \\
\hline \multirow{2}{*}{$\begin{array}{l}\text { Pt }(2045) \\
r_{o}=2.766\left[10^{-10} \mathrm{~m}\right] \\
D / k_{B}=11400.70[\mathrm{~K}] \\
b=11.65 ; a=3.05[36]\end{array}$} & $\begin{array}{c}300 \\
1000\end{array}$ & $\begin{array}{l}1.00800 \\
1.02737\end{array}$ & $\begin{array}{l}2560.72 \\
2486.63\end{array}$ & $\begin{array}{l}54.64 \\
55.57\end{array}$ & $\begin{array}{c}99.91 \\
110.52\end{array}$ & $\begin{array}{l}7.50 \\
9.00\end{array}$ & $\begin{array}{l}1.0452 \\
1.1530\end{array}$ \\
\hline & \multicolumn{2}{|c|}{$\begin{array}{l}\text { Experiments } \\
\text { and (theories) }\end{array}$} & $\begin{array}{c}2480-2490[1],(2730-2980)[1], 2480[2,3] \\
(1848-2734)[2],(2601)[3],(1680-2910)[5] \\
2490 \pm 260[8],(1810-2730)[8], 1950 \pm 15[56] \\
1940[57]\end{array}$ & & $\begin{array}{l}130[57] \\
(\mathbf{9 7})[59]\end{array}$ & & \\
\hline
\end{tabular}

ла, а тонкими линиями 2, 4, 6 - для нанокристалла при температурах $T=2000,300,10 \mathrm{~K}$ соответственно.

На рис. 1 показана барическая зависимость удельной поверхностной энергии (в $10^{-3} \mathrm{~J} / \mathrm{m}^{2}$ ) грани (100) ГЦК-Rh. Как видно из полученных зависимостей $\sigma(P)$, при $P=0$ величина $\sigma$ уменьшается с уменьшением $N$ тем заметнее, чем выше температура. Но при низких температурах и высоких давлениях на изотерме имеются две Р-точки, в которых зависимости $\sigma(P)$ для макро- и нанокристаллов пересекаются. Поэтому в Р-точке удельная поверхностная энергия не зависит от размера нанокристалла: $\sigma(N)=\sigma(\infty)$. Впервые такие Р-точки были 
Таблица 4. Значения поверхностных свойств рассчитанные для макрокристаллов ОЦК-металлов при $P=0$. (Для каждого кристалла в первой строке представлены расчеты при $T=300 \mathrm{~K}$, во второй при $T=1000 \mathrm{~K}$. Для тугоплавких металлов в третьей строке представлены расчеты при $T=2500 \mathrm{~K}$. В нижней строке в скобках представлены известные из литературы экспериментальные и теоретические данные)

\begin{tabular}{|c|c|c|c|c|c|c|c|}
\hline $\begin{array}{l}\left.\text { Metal }\left(T_{m}(P=0) / \mathrm{K}\right)[35]\right) \\
\text { Parameters of potential }(19)\end{array}$ & $\begin{array}{l}T \\
\mathrm{~K}\end{array}$ & $v / v_{o}$ & $\begin{array}{c}\sigma(100) \\
10^{-3} \mathrm{~J} / \mathrm{m}^{2}\end{array}$ & $\begin{array}{c}-\sigma^{\prime}(T)_{v} \\
\mathrm{mkJ} /\left(\mathrm{m}^{2} \mathrm{~K}\right)\end{array}$ & $\begin{array}{c}-\sigma^{\prime}(T)_{P} \\
\mathrm{mkJ} /\left(\mathrm{m}^{2} \mathrm{~K}\right)\end{array}$ & $\begin{array}{c}\sigma^{\prime}(P)_{T} \\
\mathrm{~mJ} /\left(\mathrm{m}^{2} \mathrm{GPa}\right)\end{array}$ & $\Delta_{p}$ \\
\hline \multirow{2}{*}{$\begin{array}{l}\alpha-\mathrm{Fe}(1185 \mathrm{bcc}-\mathrm{fcc}) \\
r_{o}=2.4500\left[10^{-10} \mathrm{~m}\right] \\
D / k_{B}=12653.77[\mathrm{~K}] \\
b=7.71 ; a=3.12[36]\end{array}$} & $\begin{array}{c}300 \\
1000\end{array}$ & $\begin{array}{l}1.01199 \\
103932\end{array}$ & $\begin{array}{l}2269.40 \\
2180.37\end{array}$ & $\begin{array}{l}61.70 \\
65.92\end{array}$ & 115.27 & 10.08 & $\begin{array}{l}1.0435 \\
11482\end{array}$ \\
\hline & \multicolumn{2}{|c|}{$\begin{array}{l}\text { Experiments } \\
\text { and (theories) }\end{array}$} & $\begin{array}{c}2420-2480[1],(2220-2920)[1] \\
(2520(0 \mathrm{~K})-2110(1000 \mathrm{~K}))[4], 2410[5] \\
(2220-3120)[5], 1910 \pm 190[56], 2040[57]\end{array}$ & & $(410)[4]$ & & \\
\hline \multirow{2}{*}{$\begin{array}{l}\mathrm{V}(2190) \\
r_{o}=2.6060\left[10^{-10} \mathrm{~m}\right] \\
D / k_{B}=15622.48[\mathrm{~K}] \\
b=7.52 ; a=3.08[36]\end{array}$} & $\begin{array}{c}300 \\
1000\end{array}$ & $\begin{array}{l}1.00999 \\
1.03195\end{array}$ & $\begin{array}{l}2484.13 \\
2406.07\end{array}$ & $\begin{array}{l}53.78 \\
58.49\end{array}$ & $\begin{array}{l}100.65 \\
117.02\end{array}$ & $\begin{array}{l}10.96 \\
12.68\end{array}$ & $\begin{array}{l}1.0345 \\
1.1170\end{array}$ \\
\hline & \multicolumn{2}{|c|}{$\begin{array}{l}\text { Experiments } \\
\text { and (theories) }\end{array}$} & $\begin{array}{c}2560-2620[1],(3030-3260)[1] \\
2550[3],(2462)[3],(1961)[9], 1925[57]\end{array}$ & & $120[57]$ & & \\
\hline \multirow{4}{*}{$\begin{array}{l}\mathrm{Nb}(2740) \\
r_{o}=2.8540\left[10^{-10} \mathrm{~m}\right] \\
D / k_{B}=21765.36[\mathrm{~K}] \\
b=9.34 ; a=2.53[36]\end{array}$} & 300 & 1.00800 & 2896.74 & 47.37 & 95.33 & 11.70 & 1.0295 \\
\hline & 1000 & 1.02651 & 2825.51 & 49.25 & 105.86 & 13.37 & 1.0993 \\
\hline & 2500 & 1.07380 & 2655.51 & 47.99 & 121.49 & 18.08 & 1.2565 \\
\hline & \multicolumn{2}{|c|}{ Experiments } & $\begin{array}{l}2660-2700[1],(2860-2990)[1] \\
2655-2700[2,3],(2795-2860)[2],(3113)[3] \\
(1871)[9], 2210 \pm 54[56], 2150[57]\end{array}$ & & $170[57]$ & & \\
\hline \multirow{4}{*}{$\begin{array}{l}\mathrm{Ta}(3287) \\
r_{o}=2.8560\left[10^{-10} \mathrm{~m}\right] \\
D / k_{B}=23608.03[\mathrm{~K}] \\
b=7.74 ; a=3.16[36]\end{array}$} & 300 & 1.00601 & 3143.38 & 48.09 & 87.93 & 11.15 & 1.0234 \\
\hline & 1000 & 1.01987 & 3079.07 & 49.47 & 94.38 & 12.27 & 1.0785 \\
\hline & 2500 & 1.05349 & 2931.40 & 48.56 & 102.54 & 15.19 & 1.2020 \\
\hline & \multicolumn{2}{|c|}{$\begin{array}{l}\text { Experiments } \\
\text { and (theories) }\end{array}$} & $\begin{array}{c}2900-3150[1,2],(3100-4050)[1] \\
(1790-3097)[2], 3150[3],(3144)[3] \\
(2530)[9], 2480 \pm 70[56], 2414[57]\end{array}$ & & $170[57]$ & & \\
\hline \multirow{2}{*}{$\begin{array}{l}\mathrm{Cr}(2163) \\
r_{o}=2.4850\left[10^{-10} \mathrm{~m}\right] \\
D / k_{B}=12222.45[\mathrm{~K}] \\
b=6.40 ; a=4.41[36]\end{array}$} & $\begin{array}{c}200 \\
1000\end{array}$ & $\begin{array}{l}1.00800 \\
1.02963\end{array}$ & $\begin{array}{l}2138.37 \\
2058.52\end{array}$ & $\begin{array}{l}41.29 \\
63.62\end{array}$ & $\begin{array}{c}66.87 \\
109.74\end{array}$ & $\begin{array}{l}8.42 \\
9.92\end{array}$ & $\begin{array}{r}1.0270 \\
1.13276\end{array}$ \\
\hline & & & $\begin{array}{c}2300-2350[1],(2830-3980)[1], 2300[3], \\
(2054)[3],(2017)[9], 2090 \pm 20[56], 2066[57]\end{array}$ & & $140[57]$ & & \\
\hline \multirow{4}{*}{$\begin{array}{l}\text { Mo }(2893) \\
r_{o}=2.7210\left[10^{-10} \mathrm{~m}\right] \\
D / k_{B}=19972.52[\mathrm{~K}] \\
b=8.05 ; a=3.93[36]\end{array}$} & 300 & 1.00601 & 2926.02 & 49.58 & 82.50 & 8.25 & 1.0286 \\
\hline & 1000 & 1.01884 & 2862.81 & 54.15 & 93.98 & 9.18 & 1.0960 \\
\hline & 2500 & 1.05051 & 2715.23 & 53.48 & 102.72 & 11.61 & 1.2482 \\
\hline & \multicolumn{2}{|c|}{$\begin{array}{l}\text { Experiments } \\
\text { and (theories) }\end{array}$} & $\begin{array}{c}2910-3000[1-3],(3810-3840)[1] \\
(3661-3837)[2],(2943)[3], 2070[5],(3180-3840)[5] \\
(2306)[9], 2630 \pm 50[56], 2582[57]\end{array}$ & & $180[57]$ & & \\
\hline \multirow{4}{*}{$\begin{array}{l}\mathrm{W}(3695) \\
r_{o}=2.7380\left[10^{-10} \mathrm{~m}\right] \\
D / k_{B}=25710.94[\mathrm{~K}] \\
b=8.82 ; a=3.44[36]\end{array}$} & 300 & 1.00499 & 3728.64 & 51.55 & 89.74 & 8.60 & 1.0237 \\
\hline & 1000 & 1.01629 & 3662.26 & 53.87 & 97.54 & 9.45 & 1.0799 \\
\hline & 2500 & 1.04339 & 3510.00 & 53.16 & 105.55 & 11.59 & 1.2051 \\
\hline & \multicolumn{2}{|c|}{$\begin{array}{l}\text { Experiments } \\
\text { and (theories) }\end{array}$} & $\begin{array}{c}3270-3680[1-3],(3900-4640)[1],(4403-4635)[2] \\
\quad(3764)[3],(3020)[9], 2210 \pm 22[56], 2653[57]\end{array}$ & & $170[57]$ & & \\
\hline
\end{tabular}

обнаружены в [30] при изучении свойств ОЦК-Fе. Потом параметры Р-точек были изучены для ОЦК-Та в [19], ОЦК-Nb в [62], ОЦК-W в [63], и для сплава замещения ГЦК-Аu-Fe в [31]. С ростом температуры эти Р-точки сближаются, и при высоких температурах Р-точек на изотерме уже нет. В области, оконтуренной Р-точками, величина $\sigma$ возрастает при изотермо-изобарическом уменьшении размера нанокристалла. Такое поведение функции $\sigma(P, N)$ обусловлено тем, что при низких температура и высоких давлениях поверхностное давление сжимает нанокристалл, что и приводит к появлению первой Р-точки, и к неравенству $\sigma(N)>\sigma(\infty)$. С ростом давления функция $\sigma$ для нанокристалла уменьшается заметнее, чем для макрокристалла, что и приводит к образованию второй Р-точки на изотерме. С ростом температуры поверхностное давление уменьшается, что и приводит к исчезновению области с Р-точками.

На рис. 2 показаны барические зависимости для $\sigma^{\prime}(T)_{v}$ - изохорной (4 верхние спадающие линии) и $\sigma^{\prime}(T)_{P}$ - изобарной (4 нижние возрастающие кривые) производных удельной поверхностной энергии по температуре (в $10^{-6} \mathrm{~J} /\left(\mathrm{m}^{2} \cdot \mathrm{K}\right)$ ) для ГЦК-Rh. Как 
видно из рис. 2, при низких давлениях выполняется $\left|\sigma^{\prime}(T)_{\nu}\right|<\left|\sigma^{\prime}(T)_{P}\right|$ и функция $\left|\sigma^{\prime}(T)_{\nu}\right|$ возрастает при изобарическом росте температуры намного меньше чем функция $\left|\sigma^{\prime}(T)_{P}\right|$. При $T \rightarrow 0 \mathrm{~K}$ выполняется $\sigma^{\prime}(T)_{P} \rightarrow \sigma^{\prime}(T)_{v} \rightarrow-0$, поэтому изотермы этих функций при $T=10 \mathrm{~K}$ практически равны нулю. Функция $\left|\sigma^{\prime}(T)_{\nu}\right|$ возрастает при изотермическом росте давления, а функция $\left|\sigma^{\prime}(T)_{P}\right|$ уменьшается. Это приводит к тому,

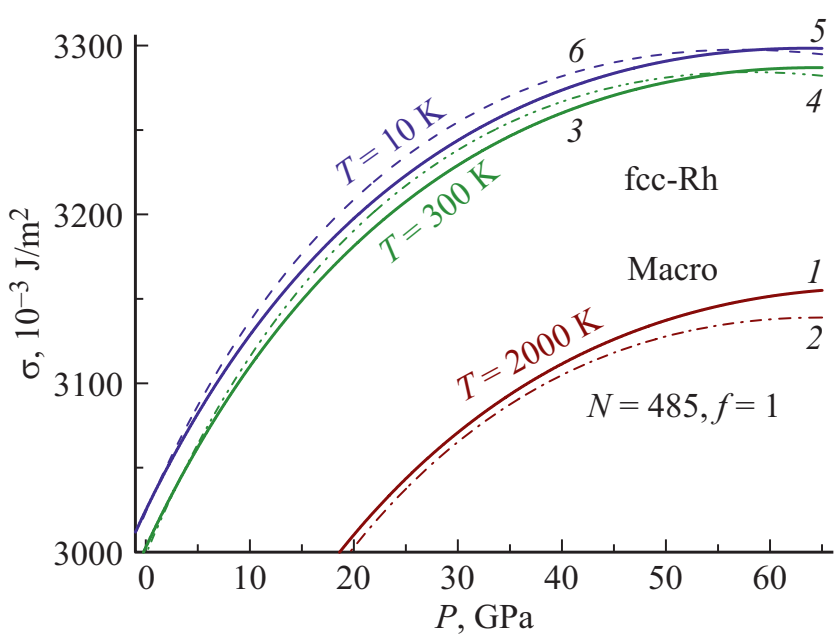

Рис. 1. Барическая зависимость удельной поверхностной энергии грани (100) для ГЦК-Rh вдоль изотерм $2000 \mathrm{~K}$ (кривые 1 и 2), 300 (кривые 3 и 4) и $10 \mathrm{~K}$ (кривые 5 и 6). Сплошные толстые кривые 1,3,5 - результаты для макрокристалла, тонкие кривые 2, 4, 6 - результаты для нанокристалла кубической формы из 485 атомов.

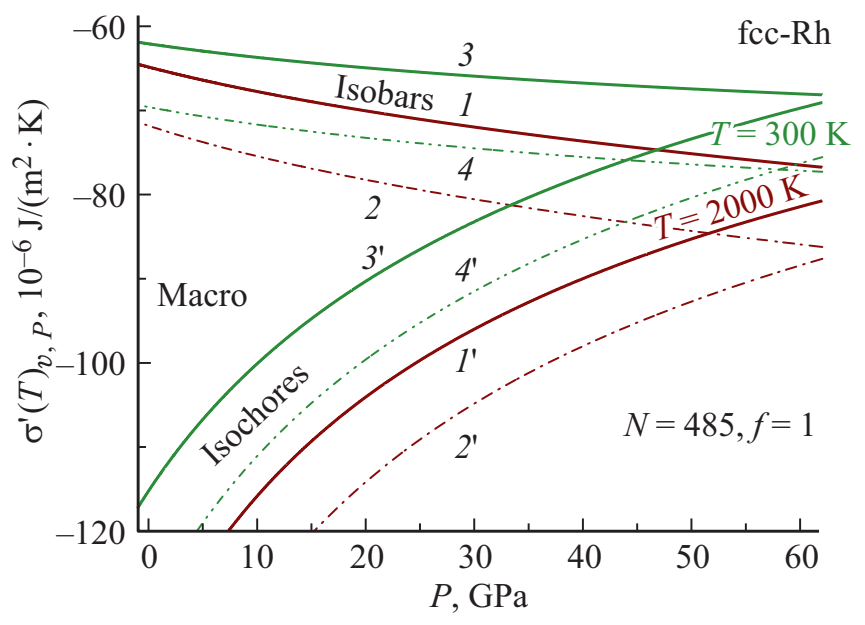

Рис. 2. Барическая зависимость для $\sigma^{\prime}(T)_{v}-$ изохорной (спадающие линии $1-4$ ) и для $\sigma^{\prime}(T)_{P}-$ изобарной (возрастающие кривые $1^{\prime}-4^{\prime}$ ) производных удельной поверхностной энергии по температуре для ГЦК-Rh. Кривые 1,2 и $1^{\prime}, 2^{\prime}$ изотермы $2000 \mathrm{~K}$, кривые 3,4 и $3^{\prime}, 4^{\prime}$ изотермы $300 \mathrm{~K}$. Изотермы $10 \mathrm{~K}$ практически равны нулю. Сплошные толстые кривые $1,1^{\prime}, 3,3^{\prime}$ - результаты для макрокристалла, тонкие кривые $2,2^{\prime}, 4,4^{\prime}$ - результаты для нанокристалла кубической формы из 485 атомов.

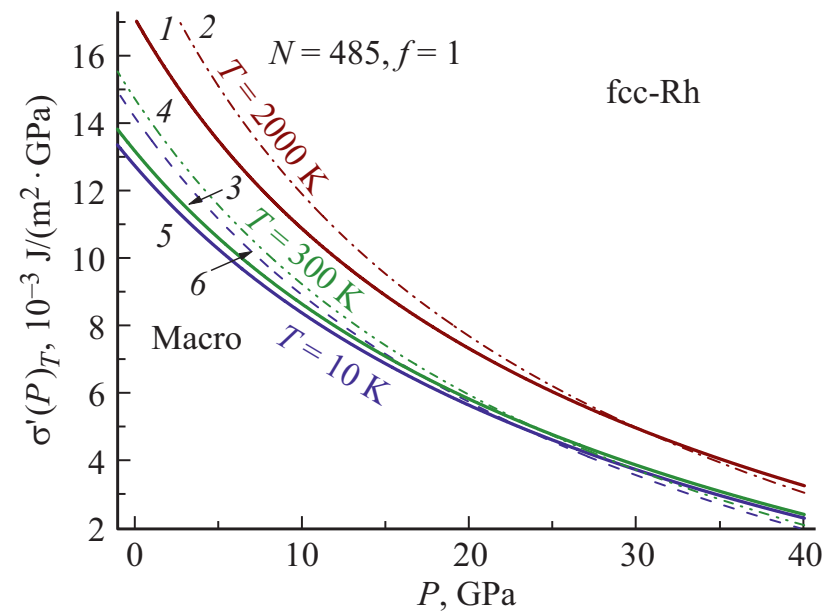

Рис. 3. Барическая зависимость производной удельной поверхностной энергии грани (100) по давлению для ГЦК-Rh вдоль изотерм $2000 \mathrm{~K}$ (кривые 1 и 2), $300 \mathrm{~K}$ (кривые 3 и 4) и $10 \mathrm{~K}$ (кривые 5 и 6). Сплошные толстые кривые 1,3,5результаты для макрокристалла, тонкие кривые 2,4,6- peзультаты для нанокристалла кубической формы из 485 атомов.

что имеется область давлений, где эти функции практически равны.

На рис. 3 показаны барические зависимости для $\sigma^{\prime}(P)_{T}-$ изотермической производной удельной поверхностной энергии ГЦК-Rh по давлению (в $10^{-3} \mathrm{~J} /\left(\mathrm{m}^{2} \cdot \mathrm{GPa}\right)$ ) вдоль изотерм: $2000 \mathrm{~K}$ (линии 1 и 2), $300 \mathrm{~K}$ (линии 3 и 4 ) и $10 \mathrm{~K}$ (линии 5 и 6). Из рис. 3 видно, что функция $\sigma^{\prime}(P)_{T}$ увеличивается при изомерно-изобарическом росте температуры. В области низких давлений величина $\sigma^{\prime}(P)_{T}$ увеличивается при изотермо-изобарическом уменьшении $N$. Но с ростом давления картина меняется на противоположную. Таким образом для определенной температуры существует определенное давление, где величина $\sigma^{\prime}(P)_{T}$ не зависит от размера нанокристалла.

\section{8. Заключение}

Предложен метод для расчета удельной поверхностной энергии $\sigma$, изохорной и изобарной производных функции $\sigma$ по температуре, и изотермических производных функции $\sigma$ по давлению и по плотности. Показано, что метод применим как для макро-, так и для нанокристалла однокомпонентного вещества с заданным числом атомов и с определенной формой поверхности.

Самосогласованным методом определены параметры парного межатомного потенциала Ми-Леннард-Джонса для 8 ГЦК-кристаллов $(\mathrm{Cu}, \mathrm{Ag}, \mathrm{Au}, \mathrm{Al}, \mathrm{Ni}, \mathrm{Rh}, \mathrm{Pd}, \mathrm{Pt})$, и для 7 ОЦК кристаллов (Fe, V, Nb, Ta, Cr, Mo, W). Показано, что при использовании этих параметров в аналитических расчетах получаются более лучшие результаты, чем при использовании параметров потенциала (19) из других работ. 
Показано, что рассчитанные значения удельной поверхностной энергии и изобарной производной функции $\sigma$ по температуре хорошо согласуются с экспериментальными оценками известными из литературы. Впервые рассчитаны значения изохорной и изобарной производных функции $\sigma$ по температуре, изотермических производных функции $\sigma$ по давлению и по плотности при $P=0$ и при различных температурах для макрокристаллов указанных 15 металлов.

На примере ГЦК-Rh изучены изменения поверхностных свойств при уменьшении размера нанокристалла вдоль изотерм $T=10,300,2000 \mathrm{~K}$. Показано, что имеются оконтуренные Р-точками $P-T$-области, в которых функция $\sigma$ возрастает при изоморфно-изотермо-изобарическом уменьшении размера нанокристалла.

Показано, что при изотермическом росте давления функция $\left|\sigma^{\prime}(T)_{\nu}\right|$ возрастает, а функция $\left|\sigma^{\prime}(T)_{P}\right|$ уменьшается. Это приводит к тому, что имеется область давлений, где эти функции практически равны. Для определенной температуры также существует определенное давление, где величина $\sigma^{\prime}(P)_{T}$ не зависит от размера кристалла.

\section{Благодарности}

Автор выражает благодарность С.П. Крамынину, Н.Ш. Газановой, 3.М. Сурхаевой и М.М. Гаджиевой за плодотворные дискуссии и помощь в работе.

\section{Финансирование работы}

Работа выполнена при финансовой поддержке РФФИ (грант № 18-29-11013_мк).

\section{Конфликт интересов}

Автор заявляет, что у него нет конфликта интересов.

\section{Список литературы}

[1] Q. Jiang, H.M. Lu, M. Zhao. J. Phys.: Condens. Matter 16, 4, 521 (2004). DOI: 10.1088/0953-8984/16/4/001

[2] J. Wang, S.Q. Wang. Surf. Sci. 630, 216 (2014). DOI: $10.1016 /$ j.susc.2014.08.017

[3] F. Aqra, A. Ayyad. Appl. Surf. Sci. 257, 15, 6372 (2011). DOI: 10.1016/j.apsusc.2011.01.123

[4] S. Schönecker, X. Li, B. Johansson, S.K. Kwon, L. Vitos. Sci. Rep. 5, 14860 (2015). DOI: 10.1038/srep14860

[5] R. Tran, Z. Xu, B. Radhakrishnan, D. Winston, W. Sun, K.A. Persson, S.P. Ong. Scientific Data 3, 1, 1 (2016). DOI: $10.1038 /$ sdata.2016.80

[6] S. De Waele, K. Lejaeghere, M. Sluydts, S. Cottenier. Phys. Rev. B 94, 23, 235418 (2016). DOI: $10.1103 /$ PhysRevB.94.235418

[7] T. Cheng, D. Fang, Y. Yang. Appl. Surf. Sci. 393, 364 (2017). DOI: 10.1016/j.apsusc.2016.09.147

[8] A. Patra, J.E. Bates, J. Sun, J.P. Perdew. Proc. Nat. Acad. Sci. 114, 44, E9188 (2017). DOI: 10.1073/pnas.1713320114
[9] V.P. Bokarev, G.Y. Krasnikov. Surf. Sci. 668, 73 (2018). DOI: 10.1016/j.susc.2017.10.020

[10] X. Zhang, W. Li, H. Kou, J. Shao, Y. Deng, X. Zhang, J. Ma, Y. Li, X. Zhang. J. Appl. Phys. 125, 18, 185105 (2019). DOI: $10.1063 / 1.5090301$

[11] И.Г. Шебзухова, Л.П. Арефьева. Журнал Технической Физики 89, 2, 306 (2019). [I.G. Shebzukhova, L.P. Aref'eva. Tech. Phys. 64, 2, 274 (2019).] DOI: $10.1134 / \mathrm{S} 1063784219020208$

[12] M. Xing, A.D. Pathak, S. Sanyal, Q. Peng, X. Liu, X. Wen. Appl. Surf. Sci. 509, 144859 (2020). DOI: $10.1016 /$ j.apsusc.2019.144859

[13] Z. Tang, Y. Chen, W. Ye. Crystals 10, 4, 329 (2020). DOI: $10.3390 /$ cryst10040329

[14] M. Kappeler, A. Marusczyk, B. Ziebarth. Materialia 12, 100675 (2020). DOI: 10.1016/j.mtla.2020.100675

[15] B. Beeler, A. Casagranda, L. Aagesen, Y. Zhang, S. Novascone. J. Nucl. Mater. 540, 152271 (2020). DOI: $10.1016 /$ j.jnucmat.2020.152271

[16] Л.Д. Ландау, Е.М. Лифшиц. Статистическая физика. Наука, M. (1976). Ч. 1. 584 c. [L.D. Landau, E.M. Lifshitz. Statistical Physics. Pergamon Press, Oxford, U.K. (1980). V. I.].

[17] Э.А. Мелвин-Хьюз. Физическая химия. Изд-во ИЛ, М. (1962), 1148 c. [E.A. Moelwyn-Hughes. Physical Chemistry. Pergamon Press, London (1961).].

[18] М.Н. Магомедов. ФТТ 46, 5, 924 (2004). [M.N. Magomedov. Phys. Solid State 46, 5, 954 (2004).]. DOI: 10.1134/1.1744976

[19] М.Н. Магомедов. Поверхность. Рентген., синхротр., и нейтрон. исслед. 11, 88 (2020). [M.N. Magomedov. J. Surf. Investigation. X-ray, Synchrotron Neutron Tech. 14, 6, 1208 (2020).]. DOI: $10.1134 / \mathrm{S} 1027451020060105$

[20] М.Н. Магомедов. Теплофизика высоких температур 43, 6, 870 (2005). [M.N. Magomedov. High Temperature 43, 6, 870 (2005).] DOI: 10.1007/s10740-005-0134-0

[21] М.Н. Магомедов. ЖТФ 83, 6, 155 (2013). [M.N. Magomedov. Tech. Phys. 58, 6, 927 (2013).]. DOI: $10.1134 / \mathrm{S} 1063784213060212$

[22] Э.Л. Нагаев. Успехи физ. наук 162, 9, 49 (1992). [E.L. Nagaev. Sov. Phys. Usp. 35, 9, 747-782 (1992).]. DOI: $10.3367 /$ UFNr.0162.199209b.0049

[23] М.Н. Магомедов. Кристаллография 62, 3, 487 (2017). [M.N. Magomedov, Crystallogr. Rep. 62, 3, 480 (2017).]. DOI: $10.1134 / \mathrm{S} 1063774517030142$

[24] M.N. Magomedov. J. Mol. Liquids 285, 106 (2019). DOI: $10.1016 /$ j.molliq.2019.04.032

[25] Л. Жирифалько. Статистическая физика твердого тела. Мир, М. (1975), 383 с. [L.A. Girifalco. Statistical Physics of Materials, J. Wiley and Sons Ltd., N.Y. (1973).].

[26] М.Н. Магомедов. ЖТФ 83, 9, 56 (2013). [M.N. Magomedov. Tech. Phys. 58, 9, 1297 (2013).]. DOI: $10.1134 / \mathrm{S} 106378421309020 \mathrm{X}$

[27] М.Н. Магомедов. Рос. нанотехнологии 14, 1-2, 19 (2019). [M.N. Magomedov. Nanotechnol. Rus. 14, 1-2, 21 (2019).]. DOI: 10.1134/S1995078019010063

[28] М.Н. Магомедов. Поверхность. Рентген., синхротр., и нейтрон. исслед. 11, 107 (2013). [M.N. Magomedov. J. Surf. Investigation. X-ray, Synchrotron Neutron Tech. 7, 6, 1114 (2013).]. DOI: 10.1134/S1027451013060104

[29] М.Н. Магомедов. ЖТФ 86, 5,84 (2016). [M.N. Magomedov. Tech. Phys. 61, 5, 722 (2016).]. DOI: $10.1134 / \mathrm{S} 1063784216050145$ 
[30] М.Н. Магомедов. Поверхность. Рентген., синхротр., и нейтрон. исслед. 2, 103 (2018). [M.N. Magomedov. J. Surf. Investigation. X-ray, Synchrotron Neutron Tech. 12, 1, 185 (2018).]. DOI: 10.1134/S1027451018010299

[31] М.Н. Магомедов. ФТТ 62, 12, 2034 (2020). [M.N. Magomedov. Phys.Solid State 62, 12, 2280 (2020).]. DOI: $10.1134 / \mathrm{S} 1063783420120197$

[32] М.Н. Магомедов. Теплофизика высоких температур 44, 4, 518 (2006). [M.N. Magomedov. High Temperature 44, 4, 513 (2006).]. DOI: 10.1007/s10740-006-0064-5

[33] М.Н. Магомедов. ФТТ 62, 7, 998 (2020). [M.N. Magomedov. Phys. Solid State 62, 7, 1126 (2020).]. DOI: $10.1134 / \mathrm{S} 1063783420070136$

[34] S. Zhen, G.J. Davies. Phys. Status Solidi A 78, 2, 595 (1983). DOI: $10.1002 /$ pssa.2210780226

[35] М.Н. Магомедов. Изучение межатомного взаимодействия, образования вакансий и самодиффузии в кристаллах, Физматлит, М. (2010). 544 с.

[36] P. Janthon, S. Luo(Andy), S.M. Kozlov, F. Viñes, J. Limtrakul, D.G. Truhlar, F. Illas. J. Chem. Theory Comput. 10, 9, 3832 (2014). DOI: $10.1021 /$ ct500532v

[37] Л.В. Альтшулер, С.Б. Кормер, А.А. Баканова, Р.Ф. Трунин. ЖЭТФ 38, 3, 790 (1960). [L.V. Al’Tshuler, S.B. Kormer, A.A. Bakanova, R.F. Trunin. Sov. Phys. JETP 11, 3, 573 (1960).]. http://www.jetp.ac.ru/cgi-bin/dn/e_011_03_0573.pdf

[38] П.И. Дорогокупец, Т.С. Соколова, Б.С. Данилов, К.Д. Литасов. Геодинамика и тектонофизика 3, 2, 129 (2012). [P.I. Dorogokupets, T.S. Sokolova, B.S. Danilov, K.D. Litasov. Geodynamics Tectonophys. 3, 2, 129 (2012).] DOI: $10.5800 / G T-2012-3-2-0067$

[39] С.И. Новикова. Тепловое расширение твердых тел. Наука, M. (1974), $294 \mathrm{c}$

[40] J.K. Baria, A.R. Jani. Physica B: Condens. Matter 328, 3-4, 317 (2003). DOI: 10.1016/S0921-4526(02)01856-2

[41] Ч. Киттель. Введение в физику твердого тела. Наука, М. (1978). 792 c. [Ch. Kittel. Introduction to Solid State Physics, J. Wiley and Sons Ltd., N.Y. (1976).]

[42] M.M. Shukla, N.T. Padial. Rev. Brasil. Fúisica 3, 1, 39 (1973). http://sbfisica.org.br/bjp/download/v03/v03a03.pdf

[43] A. Karbasi, S.K. Saxena, R. Hrubiak. CALPHAD: Comput. Coupling Phase Diagrams Thermochem. 35, 1, 72 (2011). DOI: $10.1016 /$ j.calphad.2010.11.007

[44] M.G. Pamato, I.G. Wood, D.P. Dobson, S.A. Hunt, L. Vočadlo. J. Appl. Crystallography 51, 2, 470 (2018). DOI: $10.1107 / \mathrm{S} 1600576718002248$

[45] R.G. McQueen, S.P. Marsh. J. Appl. Phys. 31, 7, 1253 (1960). DOI: $10.1063 / 1.1735815$

[46] Z.Y. Zeng, C.E. Hu, L.C. Cai, F.Q. Jing. Physica B: Condens. Matter 407, 3, 330 (2012). DOI: 10.1016/j.physb.2011.10.047

[47] P. Kumar, N.K. Bhatt, P.R. Vyas, V.B. Gohel. Eur. Phys. J. B 89, 10, 1 (2016). DOI: 10.1140/epjb/e2016-70367-0

[48] K.V. Yusenko, S. Khandarkhaeva, T. Fedotenko, A. Pakhomova, S.A. Gromilov, L. Dubrovinsky, N. Dubrovinskaia. J. Alloys Comp. 788, 212 (2019). DOI: 10.1016/j.jallcom.2019.02.206

[49] D.R. Wilburn, W.A. Bassett. Am. Mineralogist 63, 5-6, 591 (1978). https://pubs.geoscienceworld.org/msa/ammin/articleabstract/63/5-6/591/40926

[50] Y. Shibazaki, K. Nishida, Y. Higo, M. Igarashi, M. Tahara, T. Sakamaki, H. Terasaki, Y. Shimoyama, S. Kuwabara, Y. Takubo, E. Ohtani. Am. Mineralogist 101, 5, 1150 (2016). DOI: 10.2138/am-2016-5545
[51] K. Wang, R.R. Reeber. Mater. Sci. Eng. Rep. 23, 3, 101 (1998). DOI: 10.1016/s0927-796x(98)00011-4

[52] L. Koči, Y. Ma, A.R. Oganov, P. Souvatzis, R. Ahuja. Phys. Rev. B 77, 21, 214101 (2008). DOI: 10.1103/PhysRevB.77.214101

[53] L. Ming, M.H. Manghnani. J. Appl. Phys. 49, 1, 208 (1978). DOI: $10.1063 / 1.324325$

[54] X. Huang, F. Li, Q. Zhou, Y. Meng, K.D. Litasov, X. Wang, B. Liu, T. Cui. Sci. Rep. 6, 19923 (2016). DOI: $10.1038 /$ srep19923

[55] М.Н. Магомедов. ЖТФ 80, 9, 150 (2010). [M.N. Magomedov. Tech. Phys. 55, 9, 1382 (2010).]. DOI: $10.1134 / \mathrm{S} 1063784210090240$

[56] V.K. Kumikov, Kh.B. Khokonov. J. Appl. Phys. 54, 3, 1346 (1983). DOI: $10.1063 / 1.332209$

[57] B.B. Alchagirov, T.M. Taova, Kh.B. Khokonov. Transact. JWRI. Special Issue (Jpn) 30, 287 (2001). https://repository.exst.jaxa.jp/dspace/handle/a-is/48071

[58] S.N. Zhevnenko. Metallurg. Mater. Transact. A 44, 6, 2533 (2013). DOI: 10.1007/s11661-013-1641-y

[59] С.Н. Задумкин. Докл. АН СССР 112, 3, 453 (1957). http://www.mathnet.ru/links/c97c74236a89a8ac731b 021056fa72ca/dan21559.pdf

[60] N. Mathur, T. Mane, D. Sundaram. Chem. Phys. 522, 188 (2019). DOI: 10.1016/j.chemphys.2019.03.007

[61] Электронная база данных: http://www.chem.msu.su/cgibin/tkv.pl.

[62] S.P. Kraminin. J. Phys. Chem. Solids 143, 109464 (2020). DOI: 10.1016/j.jpcs.2020.109464

[63] S.P. Kraminin. J. Phys. Chem. Solids 152, 109964 (2021). DOI: $10.1016 /$ j.jpcs.2021.109964

Редактор Т.Н. Василевская 